The Munich Re Programme: Evaluating the Economics of Climate Risks and Opportunities in the Insurance Sector

\title{
Integrated EUA and CER price modeling and application for spread option pricing
}

\author{
Pauline Barrieu and Max Fehr \\ March 2011 \\ Centre for Climate Change Economics and Policy \\ Working Paper No. 50 \\ Munich Re Programme Technical Paper No. 7 \\ Grantham Research Institute on Climate Change and \\ the Environment \\ Working Paper No. 40
}


The Centre for Climate Change Economics and Policy (CCCEP) was established by the University of Leeds and the London School of Economics and Political Science in 2008 to advance public and private action on climate change through innovative, rigorous research. The Centre is funded by the UK Economic and Social Research Council and has five inter-linked research programmes:

1. Developing climate science and economics

2. Climate change governance for a new global deal

3. Adaptation to climate change and human development

4. Governments, markets and climate change mitigation

5. The Munich Re Programme - Evaluating the economics of climate risks and opportunities in the insurance sector (funded by Munich Re)

More information about the Centre for Climate Change Economics and Policy can be found at: http://www.cccep.ac.uk.

The Munich Re Programme is evaluating the economics of climate risks and opportunities in the insurance sector. It is a comprehensive research programme that focuses on the assessment of the risks from climate change and on the appropriate responses, to inform decision-making in the private and public sectors. The programme is exploring, from a risk management perspective, the implications of climate change across the world, in terms of both physical impacts and regulatory responses. The programme draws on both science and economics, particularly in interpreting and applying climate and impact information in decision-making for both the short and long term. The programme is also identifying and developing approaches that enable the financial services industries to support effectively climate change adaptation and mitigation, through for example, providing catastrophe insurance against extreme weather events and innovative financial products for carbon markets. This programme is funded by Munich Re and benefits from research collaborations across the industry and public sectors.

The Grantham Research Institute on Climate Change and the Environment was established by the London School of Economics and Political Science in 2008 to bring together international expertise on economics, finance, geography, the environment, international development and political economy to create a worldleading centre for policy-relevant research and training in climate change and the environment. The Institute is funded by the Grantham Foundation for the Protection of the Environment, and has five research programmes:

1. Use of climate science in decision-making

2. Mitigation of climate change (including the roles of carbon markets and lowcarbon technologies)

3. Impacts of, and adaptation to, climate change, and its effects on development

4. Governance of climate change

5. Management of forests and ecosystems

More information about the Grantham Research Institute on Climate Change and the Environment can be found at: http://www.Ise.ac.uk/grantham.

This working paper is intended to stimulate discussion within the research community and among users of research, and its content may have been submitted for publication in academic journals. It has been reviewed by at least one internal referee before publication. The views expressed in this paper represent those of the author(s) and do not necessarily represent those of the host institutions or funders. 


\title{
Integrated EUA and CER price modeling and application for spread option pricing *
}

\author{
Pauline Barrieu \\ Statistics Department and Centre for the Analysis of Time Series \\ London School of Economics and Political Science \\ London, WC2A 2AE \\ P.M.Barrieu@lse.ac.uk \\ Max Fehr \\ Centre for the Analysis of Time Series \\ Grantham Research Institute on Climate Change and the Environment \\ London School of Economics and Political Science \\ London, WC2A 2AE \\ M.W.Fehr@lse.ac.uk
}

\begin{abstract}
In this paper we propose a market consistent futures price dynamics model for cap-andtrade schemes, designed in the spirit of the European Union's Emissions Trading Scheme (EU ETS). Historical price dynamics for the EU ETS suggest that, both, European emission Allowances (EUAs) and Certified Emission Reductions (CERs), certificates, which are generated through the Clean Development Mechanism (CDM) - a non-domestic offset mechanism - are significantly related. We use an equilibrium framework to demonstrate that compliance regulation singles out special joint futures price dynamics. Based on this result we propose an arbitrage free futures price model and apply it to the pricing of spread options between EUAs and CERs.
\end{abstract}

Key words: Environment, Asset Pricing, Stochastic Model Applications, Markov Processes,Economics

\section{Introduction}

To mitigate climate change, concrete targets for curbing green house gas emissions were established in the Kyoto Protocol (KP), in 1997. All Annex 1 Parties (industrialized countries and countries with economies in transition) that ratified the Kyoto Protocol are obliged to reach a

${ }^{*}$ This research was supported by the Munich Re Programme within the Centre for Climate Change Economics and Policy, London School of Economics and Poltical Science. Both authors would like to thank the carbon market group within the Grantham Research Institute on Climate Change and the Environment and, in particular, Alessandro Vitelli and Umut Cetin, for stimulating discussions. 
domestic target for Greenhouse Gas emissions, on a CO2 equivalent basis, according to collective reduction targets of an average 5,2\% below 1990 levels, by the first commitment period of 2008 to 2012 (EU ETS Phase II). To facilitate compliance, the Kyoto Protocol proposes three flexible mechanisms: Emission Trading, the Clean Development Mechanism (CDM), and Joint Implementation (JI). Both JI and CDM allow Annex 1 countries, or companies, to generate emission allowances by financing emission reduction projects in other countries. The permits generated under CDM are called Certified Emission Reductions (CERs), while the permits generated under JI are called Emission Reduction Units (ERUs).

In order to ensure that its KP emission targets are met in a cost efficient way, the EU introduced the European Emission Trading Scheme. This legally binding Cap-and-Trade System implements an emission trading scheme at company level, and covers more than 10,000 installations. For these installations (or agents), the respective governments annually allocate carbon emission allowances (EUAs) according to National Allocation Plans (NAPs), which define an emission limit (cap) for each compliance period. At the end of each year, emissions must be covered either by EUAs, or (since 2008) alternatively, CERs or ERUs restricted by an import limit, i.e. the maximum amount of offset certificates - specified in the NAP - that can be surrendered for compliance.

If agents fail to offset their emissions at the end of the year a financial penalty is due, which does not redeem the agent from covering excess emissions with the next year's allocation of allowances. To avoid these penalties, agents may either reduce their own emissions, buy/generate CERs, or purchase allowances from others. This transfer of emission credits through trading reduces the costs caused by regulation; the idea being, that agents able to easily reduce emissions, will do so, and, can then, sell their excess allowances to others for which reductions are harder to achieve.

Although the market for ERUs is still very small, taking place over-the-counter, several exchanges in Europe are now committed to trade EUAs and CERs. Today, the products listed on these markets are spot and futures contracts with quarterly and yearly maturity with physical delivery of CERs as well as Phase II and Phase III EUAs.

According to present EU ETS regulation, both CERs, ERUs and EUAs are eligible to offset the same amount of $\mathrm{CO}_{2}$ emissions. Nevertheless, their prices are not equal, mainly due to the difference in the regulation for their respective use (see e.g. [Sikorski(2008)]). This is particular to emission markets, and distinguishes them from other commodity markets. This will be further discussed in Section 2.

This work focuses on the study of arbitrage free price dynamics for CERs and EUAs for succeeding compliance periods, and shows how the compliance regulation singles out special joint futures price dynamics, referred to, later, as consistent.

Conceptually, the paper is divided into two parts. Since the regulation links together EUA and CER prices at the end of the compliance period, Section 3 starts with a deterministic, non-dynamic equilibrium analysis of the compliance strategies, and the resulting EUA and CER prices at this time point only. Based on this equilibrium, we define a consistency relation between CER prices and EUA prices for succeeding compliance periods. 
In the second part of the paper, we build on these results to propose an arbitrage free continuous-time model for EUA and CER price dynamics consistent, at the end of the compliance period, with the compliance regulation. Thus, in Section 4 we define minimal properties, and propose a general framework, to construct an emission market model. Then, we consider a subclass of models, which we term a lognormally driven emission market model; further, discussing different price simulations, and commenting on the wide range of different interdependence structures that can be generated.

Finally, in Section 5 we prove market completeness, and apply this model framework to price EUA-CER spread options. Such a contract allows the import limit (a regulatory parameter) to be traded between various agents regulated by EU ETS. For the reader's convenience, all proofs are retained in the Appendix and excluded from the main body of the paper.

The introduction finishes with a brief reference to the literature closely related to the present topic. For a more detailed literature review on emission trading, see: [Carmona et al.(2010)]. Many papers consider emission market equilibria in a deterministic setting, e.g. the seminal non-dynamic papers [Dales(1968)] and [Montgomery(1972)] as well as [Tietenberg(1985)], [Cronshaw and Kruse(1996)] and [Rubin(1996)], which provide a foundation for dynamic permit trading. Most of the mathematical finance literature on Emission Trading represents the price of EUAs as a stochastic process and can be divided into two main lines of research. The first one addresses EUA price evolution under dynamic stochastic equilibria. This is done in [Carmona et al.(2009)], [Chesney and Taschini(2008)], [Carmona and Fehr(2010)], [Carmona et al.(2008)] as well as [Carmona et al.(2010)], which consider the joint price dynamics of EUAs and forwards on products for which the manufacture causes pollution. The second line of research is on reduced form models for the EUA price dynamics in the first EU ETS test phase (Phase I) from 2005 to 2007, when the market was not linked to the CDM. This topic is covered by [Carmona and Hinz(2009)], [Cetin and Verschuere(2010)], [Bloch(2010)], [Gruell and Taschini (2010)] and [Seifert et al.(2008)].

\section{Modeling Framework and Characteristics of EU ETS}

In this section we introduce our modeling approach and present some important features that any market consistent model should capture. In subsection 2.1, we introduce some basic notation and explain the key elements of our modeling framework. Then, in subsection 2.2, we provide a summary of the compliance regulation of EU ETS; and, in subsection 2.3, we analyze what kind of properties the regulation imposes on the different permit prices. We close this section by showing that these properties cannot be obtained through calculations based upon a naive model, and emphasize the clear need for a more advanced modeling approach such as that presented in this paper.

\subsection{Modeling Framework}

It appears, based upon current regulatory frameworks, that most cap-and-trade schemes will include penalty, banking and borrowing regulations similar to the EU ETS, and, furthermore, that they will be linked to some form of non-domestic offset market (e.g. as the CDM). 
Therefore, for the reader's convenience, we base our terminology on the second Phase of the EU ETS, but the results presented here are likely to be representative of other upcoming capand-trade schemes. In this sense, Phases II and III, will refer to any two consecutive compliance periods in the respective cap-and-trade scheme; Phases II and III EUA refer to the first and second periods of allowances in this scheme, while CER refers to a permit generated in the non-domestic offset market, to which the EU ETS is linked. We focus our analysis on three futures contracts with December 2012 maturity, written on the following spot contracts: Phase II EUA, Phase III EUA and CER. Thereby, we restrict our attention to the corresponding price dynamics over the Phase II compliance period $[0, T]$, and denote the price processes by $\left(A_{t}\right)_{t \in[0, T]},\left(A_{t}^{\prime}\right)_{t \in[0, T]}$ and $\left(C_{t}\right)_{t \in[0, T]}$.

In our framework, these are the benchmark contracts, and any futures price with other maturities, as well as spot prices, can be derived by simple no-arbitrage arguments once a model for the term structure of interest rates has been specified.

As previously mentioned, the regulation links together EUA and CER prices at the end of the compliance period, $T$. Hence, we first derive an equilibrium at $T$, where deterministic prices, $A, A^{\prime}$ and $C$, are compatible with the compliance regulation, before constructing arbitrage-free price processes $\left(A_{t}\right)_{t \in[0, T]},\left(A_{t}^{\prime}\right)_{t \in[0, T]}$ and $\left(C_{t}\right)_{t \in[0, T]}$.

An initial explanation of the EUA/CER price spread in a dynamic stochastic equilibrium framework has been developed in [Carmona and Fehr(2010)]. This paper investigates the inner workings of indirectly linked emission trading schemes. However, due to the complexity of the model, it is not aimed at the pricing and hedging of derivatives. In this paper, we attempt to bridge the gap between this equilibrium model and reduced form models that are designed for their tractability and ease of price data calibration. Therefore, we focus on a very general modeling framework, providing a toolbox, that can be applied to a broad range of differing compliance situations. When applied to a specific market, this general model can be broken down into a simpler model, with less parameters, so that it is, again, easier to handle and calibrate.

\subsection{Regulation of EU ETS}

The European Emission Trading Scheme was launched in 2005, with a test period run from 2005 to 2007. The fully fledged system, with linkage to CDM, started with phase II, from 2008 to 2012, and will proceed with phase III from 2012 to 2020. The precise regulation is as follows: in February each year a certain number of allowances, specified within the NAPs, are allocated for free to each market participant. Then, in April each year, agents have to offset their emissions for the preceding year. This can be done either with allowances or with CERs, under the restriction that the total amount of CERs used, up to the end of the compliance period, is limited by the import cap. Technically, when surrendering CERs for compliance, these are exchanged for EUAs and are then canceled to offset emissions. If agents fail to offset their emissions at the end of the year a financial penalty is due, which does not redeem the agent from covering excess emissions with the next year's allocation of allowances. Moreover, excess allowances from one year may be banked to the next year, allowing agents to use allowances from preceding years, and compliance periods, to cover later emissions, i.e. banking is not only allowed during a compliance period but also between allowance periods. In particular in the 
EU ETS, all Phase II EUAs that are not offset for emissions are automatically converted to Phase III EUAs at the end of the compliance period. The opposite mechanism of banking, called borrowing, works as follows: since the allowances of various years that are within the same compliance period cannot be distinguished, and emissions for a given year do not have to be covered until the April of the subsequent year, the offset can be made by the subsequent year's allowances, which are allocated in February. However, this offset can only be made by certificates from the same trading period, and consequently borrowing is only allowed within (but not between) compliance periods. Hence, it seems very unlikely that any agents will fail to comply within a compliance period. As a consequence, without any loss of generality, we can restrict our attention to the situation where compliance has only to be reached at the end of the compliance period. As described in the previous subsection, we will, therefore, simply consider three benchmark futures contracts with the same December 2012 maturity, and, that follow the underlying spot contracts: phase II EUA, phase III EUA and CER.

Although we have attempted to stay as close as possible to the EU ETS regulation, there is one important feature that we do not cover. Namely, during Phase II, the decision was taken to allow any unused import limit quota for Phase II to be transferred to Phase III. We neglect this regulatory feature, as it was not included in the original design of EU ETS, but, rather, has been applied as a patch, partly with the aim of compensating for a lower than expected CER/ERU supply, see e.g. [Lewis and Curien(2009)].

\subsection{Impact of Regulation on EUA and CER Prices}

Although CERs and EUAs are used to offset the same amount of $\mathrm{CO}_{2}$ emissions, their prices are not equal. The main reason for this discrepancy is due to the regulatory differences for the use of CERs in EU ETS, see e.g. [Sikorski(2008)]. Indeed, let us first assume that there is no import limit, and that the CER price is lower than the EUA price. In this case, the regulated companies will then try to cover all their emissions with CERs and bank all their allowances. This will drive the CER price up and the EUA price down until both prices converge.

The main reason that this convergence does not happen with any certainty is due to the import limit. Let us assume that there is a significantly larger CER supply. Even if we make calculations that assume the full import limit of CERs, it is still possible that the CER price could stay below the EUA price. In fact, this was the case with early CER supply projections ${ }^{1}$, and explains why EUA and CER prices are prone to deviation.

However, prices for CERs and EUAs are, in contrast, closely related. Indeed, it is possible to obtain some market bounds for CERs and ongoing period's EUAs, as well as next period's EUAs. To this end, we consider only the last time point of the compliance period, and assume that next period's allowances are already spot traded (i.e they are allocated), and the compliance, just as the surrendering of next period's allowances (in case of non-compliance), have to take place instantaneously.

\footnotetext{
${ }^{1}$ For instance, in March 2008 the projected CER supply for the 2008-2012 compliance period was 2.3Gt. With the import limit of $1.4 \mathrm{Gt}$, this would have left the rest of the CER market with 900Mt of CERs, which, in turn, would have implied a rather low CER price. However, in July 2008 the CER supply projection was corrected downwards to $1.7 \mathrm{Gt}$, and, again, in March 2010, to $1.0 \mathrm{Gt}$.
} 


\subsubsection{Lower and Upper Market Bounds for EUA Price}

Since emissions can always be covered by paying the penalty, and surrendering next year's allowances, the ongoing period's EUA price is bounded from above by next period's EUA price plus the penalty. Moreover, since the banking of EUAs to the next period is allowed, the ongoing period's EUA price is bounded from below by next period's EUA price.

Besides these upper and lower bounds for the ongoing period EUA prices, the EUA price is also bounded from below by the CER price. Indeed, assuming that the CER price exceeds the EUA price, then all agents would only use EUAs for compliance. If, moreover, the number of EUAs is not enough to cover emissions, then the scarcity of EUAs drives the price up; when, first, it equals the CER price, agents become indifferent between using EUAs or CERs. However, this holds true only if emissions exceed the initial allocation.

However, there is another situation where the EUA price is bounded from below by the CER price. In some circumstances, the CER price is a lower bound for next period's EUA price, which, in turn, is a lower bound for the ceasing period's EUA price, as explained in the next paragraph.

\subsubsection{Lower Bound for Next Periods EUA Price}

To reach compliance, an agent can surrender any amount of CERs between their compliance gap and their import limit. In view of the regulation, explained in Section 2.2, any amount of CERs surrendered beyond the compliance gap is automatically converted to Phase III EUAs. Hence, by altering the amount of CERs surrendered for compliance, agents control which asset, Phase III EUAs or CERs, they carry over to the next compliance period. As it is rational to transfer as much as possible of the more valuable commodity to the next period, the next period's allowance price and the CER price should tend to converge. However, this convergence may not be complete in case that the surrendered amount reaches either its lower bound (the compliance gap) or its upper bound (the import limit).

In the case that EUA price exceeds the CER price, agents use the full import limit of CERs for compliance. On the other hand, if the CER price exceeds the EUA price, the market uses only a number of CERs corresponding to the compliance gap (if CER price stays below penalty plus next periods EUA price). However, in Phase II of the EU ETS, this compliance gap is expected to be relatively small (see Appendix D.1).

Hence, if the according amount of CERs are surrendered for compliance, there will still be so many CERs available outside the EU ETS that the price is expected to stay below next period's EUA price, and the CER price will be bounded from above by next period's EUA price.

This shows the clear need for an arbitrage free and consistent market model that fulfills these typical properties. In the present paper, we will go even further and propose a model framework, which covers all theoretically possible outcomes from the EU ETS. In particular, this framework will include all upper bound properties from above and will allow the CER and next period's EUA prices to converge with positive probability. 


\section{Partial Equilibrium}

In this section, we perform a more formal investigation into how the compliance regulation influences differing allowance and CER prices. Consistent with the common view, we maintain that realistic market prices are described by a market equilibrium. As discussed in Section 2.2, we focus on the situation where compliance takes place only at the end of the compliance period, and consider a simple and non-probabilistic equilibrium at the last time point of the compliance period. In subsection 3.1, we explain the equilibrium setting. In subsection 3.2, we derive equilibrium prices, and show that they can be determined based on the optimal compliance strategy of the representative planer problem based on an aggregated cost function. Based on this result, we assume, in subsection 3.3, a specific form of the aggregated cost function. In view of this assumption, we reformulate equilibrium prices and introduce a reparametrization of the compliance strategies, which will be used in the remainder of this paper.

\subsection{Definition of Equilibrium}

Conceptually, the price formation of EUAs and CERs are considerably different. Allowances are traded in local markets that are governed by local cap and trade schemes, while CERs are traded on a global CDM market; however, with potential links to several local cap-and-trade schemes. For simplicity, we model only one cap-and-trade scheme in relation to the global CER market. Therefore, a finite set of agents, $I$, covered by the cap and trade scheme, is distinguished from a finite set of agents, $J$, which are outside of the cap and trade scheme.

Agents in the cap and trade scheme We start by introducing the agents, $I$, that are considered to be regulated by the cap and trade scheme. Each agent, $i$, will have some emission $\mathcal{E}^{i}$. At the beginning of the compliance period, the regulator allocates $\Lambda^{i}$ allowances to each agent, $i$. If an agent is unable cover the emissions it is responsible for, then a financial penalty, $\pi$, is due for each ton of excess emissions, which, nonetheless, have to be accounted for during the next compliance period.

Under this scheme, in order to comply, each agent, $i$, can adjust its allowance position by buying or selling $\phi^{i} \in \mathbb{R}$ allowances ${ }^{2}$ on the market (typically an allowance exchange) at the price $A$.

Similarly, each agent, $i$, can buy $\gamma^{i}$ CERs, on the markets, which it surrenders for compliance. The amount of CERs that agent $i \in I$ surrenders for compliance cannot exceed the import limit $\Gamma^{i}$. Moreover, a short position of CERs cannot be submitted for compliance. Hence, the following constraint holds: $0 \leq \gamma^{i} \leq \Gamma^{i}$.

It is also assumed that agents can trade allowances for the following subsequent period on the market at a price $A^{\prime}$; the amount of allowances for the succeeding period that agent $i$ trades at time $T$, is denoted by $\varphi^{i} \in \mathbb{R}$. Since these allowances are not immediately surrendered for

\footnotetext{
${ }^{2}$ In reality the number of allowances that each agent can sell is restricted by the initial allocation, meaning that $\phi^{i} \geq-\Lambda^{i}$. However, since the number $\Lambda^{i}$ is usually much higher than the number of allowances that are bought, this constraint does not, in fact, add any value to the model. Therefore, we skip this constraint, as it only makes the proofs more cumbersome.
} 
compliance, agents are allowed to take arbitrarily long and short positions, and, hence, there are no upper or lower bounds for $\varphi^{i}$.

With the above notation, the net cumulative emissions for any agent, $i$, are given by $\mathcal{E}^{i}-\Lambda^{i}-$ $\phi^{i}-\gamma^{i}$. If this number is positive, then the agent does not comply and a penalty is levied per ton of excess emissions: meaning that agent $i$ pays a penalty of $\pi\left(\mathcal{E}^{i}-\Lambda^{i}-\phi^{i}-\gamma^{i}\right)^{+}$. Since it is a requirement that excess emissions are covered in the subsequent compliance period, a short position of $\left(\mathcal{E}^{i}-\Lambda^{i}-\phi^{i}-\gamma^{i}\right)$ allowances is carried over to the next compliance period. If, on the other hand, $\mathcal{E}^{i}-\Lambda^{i}-\phi^{i}-\gamma^{i}$ is non-positive, then agent $i$ will hold more allowances than is required for compliance, and $\mathcal{E}^{i}-\Lambda^{i}-\phi^{i}-\gamma^{i}$ of allowances are banked/converted to next period of allowances. Hence, in both situations, the amount $\mathcal{E}^{i}-\Lambda^{i}-\phi^{i}-\gamma^{i}$ of allowances is carried over to the next compliance period, irrelevant of whether this amount is positive or non-positive.

Moreover, we assume that each agent associates the net amount $\eta^{i} \equiv \Lambda^{i}+\phi^{i}+\varphi^{i}+\gamma^{i}-\mathcal{E}^{i}$ of next period's allowances, resulting from, say, banking, penalty withdrawals and trading, with a projected Profit and Loss (P\&L) function, denoted by $\mathcal{A}^{i}\left(\eta^{i}\right)$. Furthermore, it is also assumed that the allowance allocation for subsequent periods is implicitly included within the projection of the following period's P\&L.

To summarize, each agent $i \in I$ that is regulated by the cap and trade scheme has a quasilinear cost function ${ }^{3}$ given by:

$$
L^{A, A^{\prime}, C, i}\left(\phi^{i}, \varphi^{i}, \gamma^{i}\right)=\pi\left(\mathcal{E}^{i}-\Lambda^{i}-\phi^{i}-\gamma^{i}\right)^{+}-\mathcal{A}^{i}\left(-\mathcal{E}^{i}+\Lambda^{i}+\phi^{i}+\varphi^{i}+\gamma^{i}\right)+\phi^{i} A+\varphi^{i} A^{\prime}+\gamma^{i} C .
$$

Agents outside the cap and trade scheme Agents $J$, outside the cap and trade scheme, may represent countries who can use CERs to comply with their Kyoto targets, but also firms covered by other cap and trade schemes (e.g. a possible US or Japanese Scheme) or any other agents speculating in CERs. Rather than focusing on the compliance strategies, or allowance prices of these schemes, we are interested in their CER trading. We assume that every agent $j \in J$ can buy or sell $\beta^{j} \in \mathbb{R}$ CERs. Since this amount is not directly connected to any compliance strategy, it is not bounded, and agents are allowed to take any arbitrarily long and short position.

We assume that buying and selling CERs only influences the projected P\&L function of the various agents $j$, denoted by $\mathcal{C}^{j}\left(\beta^{j}\right)$. Hence, their quasilinear cost function is given by

$$
M^{C, j}\left(\beta^{i}\right)=-\mathcal{C}^{j}\left(\beta^{j}\right)+\beta^{j} C .
$$

Note that the agents $i \in I$, regulated by the cap and trade scheme, could also invest in CERs, either, for use in later compliance periods or for speculative reasons. This could be covered by adding a term similar to $H^{j}$ to the utility function $L^{A, A^{\prime}, C, i}$. However, this does not change the equilibrium outcomes; therefore, we restrict ourselves to the above setting.

Equilibrium In the following, $\mathcal{S}^{i}=\mathbb{R} \times \mathbb{R} \times\left[0, \Gamma^{i}\right]$ denotes the feasible compliance strategies of any agent $i \in I$, and by, $\mathcal{S}^{j}=\mathbb{R}$, the feasible compliance strategies of any agent $j \in J$.

\footnotetext{
${ }^{3}$ with the following convention that a negative cost is a gain.
} 
Moreover, $\mathcal{S}=\left\{(\phi, \varphi, \gamma, \beta) \mid\left(\phi^{i}, \varphi^{i}, \gamma^{i}\right) \in \mathcal{S}^{i}, \beta^{j} \in \mathcal{S}^{j}\right.$ for all $\left.i \in I, j \in J\right\}$ denotes the feasible compliance strategies for the overall market.

Following the intuition that, given the allowance and CER prices $\left(A, A^{\prime}, C\right)$, every agent aims at minimizing their own cost function, we introduce the following definition for equilibrium:

Definition 1. The triple of prices $\left(A^{*}, A^{*}, C^{*}\right) \in \mathbb{R}^{3}$, together with the compliance strategy $\left(\phi^{*}, \varphi^{*}, \gamma^{*}, \beta^{*}\right) \in \mathcal{S}$, form a competitive equilibrium, only if the following conditions are satisfied:

(i) All financial positions are in zero net supply, i.e.

$$
\sum_{i \in I} \phi^{* i}=0, \quad \sum_{i \in I} \varphi^{* i}=0, \quad \sum_{i \in I} \gamma^{* i}+\sum_{j \in J} \beta^{* j}=0
$$

(ii) Each agent $i \in I$, under the Cap-and-Trade Scheme, is satisfied by its own compliance strategy, namely:

$$
L^{A^{*}, A^{\prime *}, C^{*}, i}\left(\phi^{i *}, \varphi^{i *}, \gamma^{i *}\right) \leq L^{A^{*}, A^{\prime *}, C^{*}, i}\left(\phi^{i}, \varphi^{i}, \gamma^{i}\right)
$$

for all $\left(\phi^{i}, \varphi^{i}, \gamma^{i}\right) \in \mathcal{S}^{i}$

(iii) Each agent $j \in J$, outside the Cap-and-Trade Scheme, is satisfied by its CER trading strategy:

$$
M^{C^{*}, j}\left(\beta^{j *}\right) \leq M^{C^{*}, j}\left(\beta^{j}\right)
$$

For the equilibrium results stated in the next section, the following set of standard assumptions are required:

Assumption 1. (i) $\eta^{i} \mapsto \mathcal{A}^{i}\left(\eta^{i}\right)$ and $\beta^{j} \mapsto \mathcal{C}^{j}\left(\beta^{j}\right)$ are monotonically increasing, concave and continuously differentiable for all $i \in I$ and $j \in J$;

(ii) $\Gamma^{i}>0$ for all $i \in I$.

Remark 1. Differing from many other approaches posited in the literature reference, where equilibria for consecutive compliance periods are linked together, the modeling approach taken here, only considers the characterization of the equilibrium at the end of the first compliance period. Thus, this approach avoids the modeling of the equilibrium in any subsequent compliance period.

\subsection{Equilibrium Results}

To characterize the market equilibrium, it is helpful to introduce a representative planer with the following optimization problem:

$$
\begin{array}{cl}
\inf _{\gamma} & \pi(\mathcal{E}-\Lambda-\gamma)^{+}-\mathcal{A}(\Lambda-\mathcal{E}+\gamma)-\mathcal{C}(-\gamma) \\
\text { s.t. } & 0 \leq \gamma \leq \Gamma
\end{array}
$$

where the variable $\gamma$ is the aggregated amount of CERs surrendered for compliance, $\mathcal{E}=$ $\sum_{i \in I} \mathcal{E}^{i}$ is the aggregated emission, $\Lambda=\sum_{i \in I} \Lambda^{i}$ is the aggregated initial allowance allocation, $\Gamma=\sum_{i \in I} \Gamma^{i}$ is the aggregated import limit and

$$
\mathcal{A}(\eta)=\sup \left\{\sum_{i \in I} \mathcal{A}^{i}\left(\eta^{i}\right) \mid \sum_{i \in I} \eta^{i}=\eta\right\} \quad \mathcal{C}(\beta)=\sup \left\{\sum_{j \in J} \mathcal{C}^{j}\left(\beta^{i}\right) \mid \sum_{j \in J} \beta^{j}=\beta\right\}
$$


represents the aggregated $P \& L$ function, in, and, outside, the cap-and-trade scheme.

Notice that the representative planer problem is expressed solely in terms of the aggregated amount of CERs $\gamma$ surrendered for compliance. Moreover, because every single agent can only surrender a positive amount of CERs, and not more than $\Gamma^{i}$, the aggregated strategy is constrained by $\gamma \in[0, \Gamma]$, which defines the feasible set of the representative planer problems.

The following proposition characterizes the optimal CER compliance strategy of the representative planer.

Thus, by $o^{*} \in \mathbb{R}$, we denote the unconstrained CER strategy, which fulfills $\mathcal{C}^{\prime}\left(-o^{*}\right)=\mathcal{A}^{\prime}(\Lambda-$ $\left.\mathcal{E}+o^{*}\right)$, i.e. the point at which the marginal $\mathrm{P} \& \mathrm{~L}$ functions equate. Similarly, by $m^{*} \in \mathbb{R}$, we denote the unconstrained CER strategy, such that the marginal $P \& L$ functions differ by $\pi$, i.e. $\mathcal{C}^{\prime}\left(-m^{*}\right)=\mathcal{A}^{\prime}\left(\Lambda-\mathcal{E}+m^{*}\right)+\pi$. For later considerations, it is important to note that Assumption 1 implies that $o^{*} \leq m^{*}$, and that $o^{*}$, maximizes $\gamma \mapsto \mathcal{A}(\Lambda-\mathcal{E}+\gamma)+\mathcal{C}(-\gamma)$, which describes the aggregated projected $P \& L$ from Phase III EUAs and CERs carried over to the next compliance period.

Proposition 1. Under Assumption 1, it holds that the CER compliance strategy, given by

$$
\bar{\gamma}=0 \vee \min \left[\min \left[m^{*}, \Gamma\right], \max \left[\mathcal{E}-\Lambda, o^{*}\right]\right]
$$

, is an optimal solution to the representative planer problem (5).

Again, for simplicity, the above result is discussed by ruling out two unlikely cases. Firstly, the case of non compliance, i.e. we assume $\mathcal{E}-\Lambda<m^{*} \wedge \Gamma$; and secondly, we rule out the case $m^{*}<\Gamma$, where the CER price can exceed the Phase III EUA price by $\pi$ for some feasible compliance strategy. In this case, the interval $[0 \vee(\mathcal{E}-\Lambda), \Gamma]$ (referred to as the compliance interval) is nonempty and describes the range in which the number of CERs surrendered for compliance can be altered without becoming noncompliant. As explained in subsection 2.3.2, any amount of CERs surrendered for compliance beyond the compliance gap, $0 \vee(\mathcal{E}-\Lambda)$, is automatically converted to Phase III EUAs. As a consequence, by altering $\gamma$ in the range $[0 \vee(\mathcal{E}-\Lambda), \Gamma]$, the representative planer controls the amount of Phase III EUAs, or respective CERs, carried over to the next compliance period. Hence, within this compliance interval, $\bar{\gamma}$ is chosen to be as near as possible to $o^{*}$, in order that the greatest possible amount of the most valuable contracts, of either phase III EUA or CER, are carried over to the next period.

The next proposition takes into account the optimal compliance strategies from Proposition 1 to identify equilibrium prices.

Proposition 2. Let Assumption 1 hold true and $\bar{\gamma}$ be an optimal solution to the representative agent problem (5). Then, it holds that:

(i) The equilibrium exists.

(ii) The equilibrium allowance prices are given by

$$
\begin{aligned}
A^{\prime} & =\mathcal{A}^{\prime}(\Lambda-\mathcal{E}+\bar{\gamma}) \\
C & =\mathcal{C}^{\prime}(-\bar{\gamma}) \\
A & =A^{\prime}+\pi \mathbf{1}_{\{\mathcal{E} \geq \Lambda+\Gamma\}}+\min \left[\left(C-A^{\prime}\right)^{+}, \pi\right] \mathbf{1}_{\{\Lambda<\mathcal{E}<\Lambda+\Gamma\}} .
\end{aligned}
$$

(iii) If allowances are banked while a non-binding amount of CERs is used for compliance (i.e. $\mathcal{E}-\Lambda-\bar{\gamma}<0$ and $0<\bar{\gamma}<\Gamma$ ) then $C=A^{\prime}$ (and $A=C=A^{\prime}$ due to (ii)). Hence, whenever $o^{*} \in[0 \vee(\mathcal{E}-\Lambda) \Gamma]$, all prices glue together. 

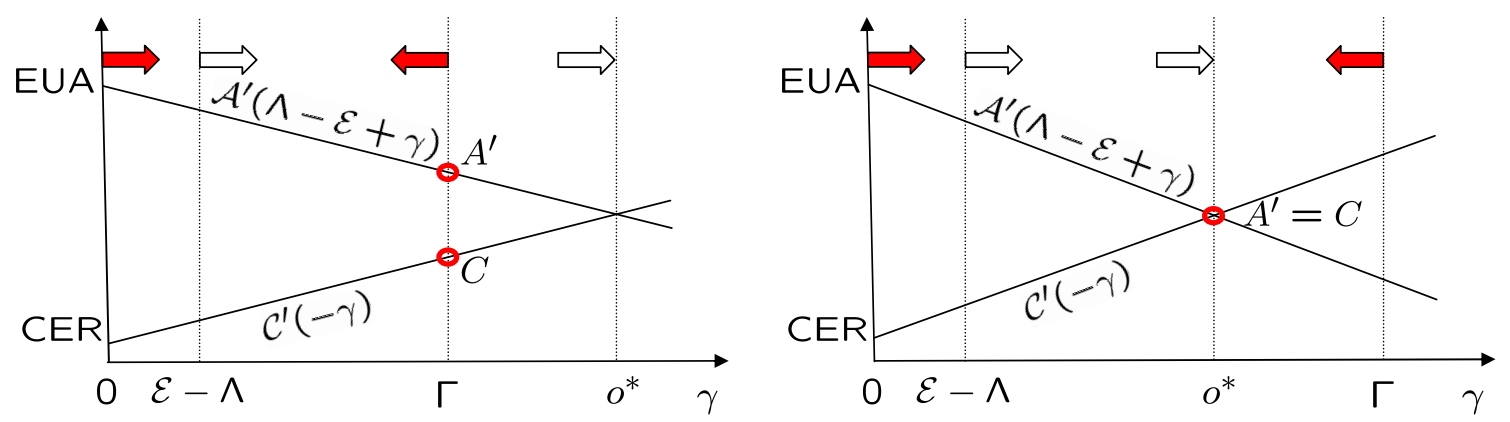

Figure 1: The first pane illustrates a situation where $o^{*}$ is larger than the import limit. Here, the optimum of the representative planer problem is attained at $\Gamma$, and it holds that $A^{\prime}>C$. On the other hand, if $o^{*}$ lies in the interior of the compliance interval, then the minimum of the representative agent problem is attained at $o^{*}$ and $A^{\prime}=C$. If these two situations are possible, it explains why the Phase II EUA price can exceed the CER price.

Proposition 2 shows that the equilibrium depends only on the aggregated P\&L functions $\mathcal{A}$ and $\mathcal{C}$, rather than on the individual ones, $\mathcal{A}^{i}$ and $\mathcal{C}^{i}$. The reason is that the various agents $i \in I$ trade Phase III EUAs until their marginal projected Phase III P\&L equate. Similarly the various agents $j \in J$ trade CERs until their marginal projected P\&Ls equate. Moreover, Proposition 2 demonstrates that given the emission $\mathcal{E}$, the CER price $C$, and the allowance prices $A$ and $A^{\prime}$, for different periods, cannot be arbitrarily chosen by the market. All these quantities have to be consistent in the sense of equation (10), and $A$ equals, either, $A^{\prime}, C$, or $A^{\prime}+\pi$. Furthermore, $A^{\prime}=C$ has to hold true in some special situations described in (iii). Two important combinations of prices and compliance strategies are illustrated in Figure 1. That prices tend to glue together is in contrast to other commodity markets, where futures can take a continuum of values (see e.g. [Hinz and Fehr(2010)]) independent of the other futures. As we will see later, in Section 5, this particularity has a strong influence on the pricing (and hedging) of options.

Interpretation To examine the interpretation of this Proposition in greater detail, we first illustrate the consistency relation given in (ii) by a standard sensitivity analysis for some key market scenarios. The proof of this Result (see Appendix) is based on duality arguments. In particular, strong duality for the representative planer problem (5) prevails, and $A$ appears as the dual variable of the Phase II trading constraint, and can be interpreted as the sensitivity of the optimal value of (5) with respect to perturbations of the trading constraint, or, equivalently, the initial allocation. This interpretation of $A$ is interesting, as we can intuit aspects of the consistency relationship (10) by studying the marginal cost of decreasing the initial allocation infinitesimally:

If, $A^{\prime}<C$ and $\Lambda<\mathcal{E}<\Lambda+\Gamma$, the full amount of EUAs is used for compliance and the compliance gap $\mathcal{E}-\Lambda$ has to be filled with CERs. Hence, a decrease in the initial allocation would have to be compensated with some CERs and $A=C$.

If, however, $A^{\prime}<C$ and $\mathcal{E}<\Lambda$, then no CERs are used for compliance. Finally, if, $A^{\prime}>$ $C$, the full amount of CERs are used for compliance. In both cases, the amount of CERs surrendered for compliance is fixed, and there is a positive amount of Phase II EUAs not used 
for compliance, and, thus, banked for the next period. Therefore, a decrease in the initial allocation leads to less EUAs being banked for the next period, and $A=A^{\prime}$.

\subsection{Reparametrization}

To achieve a higher level of tractability for the model, and, in particular, to derive a simple formula for spread option prices, as in Section 5, we introduce the following reparametrization of the model:

Let $b \equiv \Lambda+\Gamma-\mathcal{E}$ be the amount of allowances carried over from the first period to the consecutive compliance period, when the full amount $\Gamma$ of CERs is used for compliance. Note that the parameter $b$ is not necessarily non-negative. Indeed, when the total emissions, $\mathcal{E}$, exceeds $\Lambda+\Gamma$, the agents carry over a negative position $b<0$ of allowances to the next compliance period, because excess emissions have to be covered in the consecutive compliance period. In the successive section, it will be important to notice that prices glue together, i.e. $A^{\prime}=C=A$, whenever $\Gamma-o^{*} \in[0, b \wedge \Gamma]$. This is a direct consequence of Proposition 2, assertion (iii).

We also introduce the parameter $x \equiv \Gamma-\gamma$, i.e. $x$ is the remaining import limit when $\gamma$ CERs are surrendered for compliance.

Note that, with this notation, the amount of allowances carried over to the consecutive period is given by $b-x$. As $\gamma$, the new parameter $x$ is constrained by $0 \leq x \leq \Gamma$.

From Proposition 2, the equilibrium only depends on the aggregated projected P\&L functions $\mathcal{A}$ and $\mathcal{C}$, rather than every individual $\mathcal{A}^{i}$ and $\mathcal{C}^{j}$. Therefore, we will focus on the aggregated level, and consider an exponential form for the marginal aggregated projected $\mathrm{P} \& \mathrm{~L}$ functions, given as follows. For some fixed constants $a^{\prime}, c, p, q>0$, let us define

$$
\mathcal{A}^{\prime}: \mathbb{R} \rightarrow \mathbb{R}_{+} \quad \eta \mapsto a^{\prime} \exp (p(\Lambda+\Gamma-\mathcal{E}-\eta)) \quad \text { and } \quad \mathcal{C}^{\prime}: \mathbb{R} \rightarrow \mathbb{R}_{+} \quad \beta \mapsto c \exp (-q(\Gamma+\beta)) .
$$

Hence, it holds that $\left.\mathcal{A}^{\prime}(\Lambda+\Gamma-\mathcal{E}-x)\right)=a^{\prime} \exp (p x)$ and $\mathcal{C}^{\prime}(-(\Gamma-x))=c \exp (-q x)$.

Since $a^{\prime}=\mathcal{A}^{\prime}(\Lambda+\Gamma-\mathcal{E})$, it is natural that the emission $\mathcal{E}$ has an influence on $a^{\prime}$ (and in the next section we will model them as correlated random variables). Moreover, following the intuition of Section 2.3, it will be natural for the supply of CERs to have an influence on the CER price. With this reparametrization, this is done implicitly through the parameter $c$. For instance, if the CER supply is high, then $c$ should be small. On the other hand, if the CER supply is tight, $c$ should be large.

For these projected $\mathrm{P} \& \mathrm{~L}$ functions and parameters, the equilibrium result can be rewritten as follows:

Proposition 3. Let Assumption 1, part (iii), hold true. Then, it holds that:

(i) For some fixed $p>0, q>0$, and for every $a^{\prime}>0, c>0$ and $b \in \mathbb{R}$, the equilibrium allowance and CER prices are given by

$$
\begin{aligned}
A^{\prime}\left(a^{\prime}, c, b\right) & =a^{\prime} \exp \left(p x^{*}\left(a^{\prime}, c, b\right)\right) \\
C\left(a^{\prime}, c, b\right) & =c \exp \left(-q x^{*}\left(a^{\prime}, c, b\right)\right) \\
A\left(a^{\prime}, c, b\right) & =A^{\prime}\left(a^{\prime}, c, b\right)+\pi \mathbf{1}_{\{b<0\}}+\min \left[\left(C\left(a^{\prime}, c, b\right)-A^{\prime}\left(a^{\prime}, c, b\right)\right)^{+}, \pi\right] \mathbf{1}_{\{0 \leq b \leq \Gamma\}} .
\end{aligned}
$$


with

$$
x^{*}\left(a^{\prime}, c, b\right)=\max \left[\max \left[\Gamma \wedge m^{*}\left(a^{\prime}, c\right), 0\right], \min \left[\Gamma, b, \frac{\ln \left(c / a^{\prime}\right)}{p+q}\right]\right]
$$

and $m^{*}\left(a^{\prime}, c\right)$ such that $c \exp \left(-q m^{*}\right)=a^{\prime} \exp \left(p m^{*}\right)+\pi$.

(ii) For fixed $A^{\prime}>0, C>0, p>0, q>0$ and $b \in \mathbb{R}$ there exist $a^{\prime}>0, c>0$ such that

$$
A^{\prime}=A^{\prime}\left(a^{\prime}, c, b\right) \quad C=C\left(a^{\prime}, c, b\right) .
$$

The latter result implies that any pair of Phase III EUA and CER prices can be represented as parameterized functions of the pair of $a^{\prime}$ and $c$. Hence, at the end of the compliance period, any market prices $A^{\prime}$ and $C$ can be explained by such an equilibrium result.

Remark 2. Notice that $x^{*}$ is continuous in $c, a^{\prime}, b$. And $A$ is continuous in $c, a^{\prime}$, but not in $b$.

Note that from the above proposition, and, in particular, equations (11)-(14), the proposed framework will naturally lead to simple spread option prices, as will be seen in Section 7: Indeed, $A^{\prime}>C$ implies that $A^{\prime}=a^{\prime}$ and $C=c$, whilst $A^{\prime} \leq C$ implies that $a^{\prime} \leq c$. Later, $a^{\prime}$ and $c$ will be replaced by lognormally distributed random variables, which clearly lead to spread option prices similar to Margrabes formula. This will be demonstrated in Section 5.

\section{Arbitrage Free Emission Market Model}

In this Section, we introduce and discuss the main properties of, an arbitrage-free emission market model. In subsection 4.1, we define and construct such a model with some minimums, which are consistent with the equilibrium result of the previous section. In subsection 4.2, we introduce a specific subclass of models, which will be at the core of our analysis for the remainder of the paper. Furthermore, we discuss the interdependence structure between our benchmark futures in subsection 4.3. Moreover, in subsection 4.4, we show that, when applied to a specific market, the general model can be broken down into a simpler model, with less parameters, so that it is easier to handle and calibrate.

\subsection{Arbitrage Free Emission Market Model - General Definition}

This section defines an arbitrage-free emission market model, which is consistent with the equilibrium obtained in Proposition 2. More precisely, the market's emission, CER, Phase II EUA and Phase III EUA prices should be consistent.

In the following section, uncertainty is represented by a filtered probability space $(\Omega, \mathcal{F}, \mathbb{F}=$ $\left.\left(\mathcal{F}_{t}\right)_{t \in[0, T]}, \mathbb{P}\right)$, where $\mathbb{P}$ is a prior probability measure, and $\mathbb{F}$ is a filtration (satisfying the usual regularity conditions) that represents the information available to the agents at any time. We will assume that for every $0 \leq t \leq T$, that $\mathcal{F}_{t}$ includes, in particular, $\sigma\left(A_{s}, A_{s}^{\prime}, C_{s}, 0 \leq s \leq t\right)$, and $\mathcal{F}_{T}$ includes $\sigma\left(\mathcal{E}_{T}\right)$. In other words, at any time $t$, the agents observe the market prices, and at time $T$, they also observe the market's total emission, given by the random variable $\mathcal{E}_{T} \in L^{0}\left(\mathcal{F}_{T}\right)$. 
This leads us to the following Definition of an arbitrage free emission market:

Definition 2. We say that the futures price processes $\left(A_{t}\right)_{t \in[0, T]},\left(A_{t}^{\prime}\right)_{t \in[0, T]}$ and $\left(C_{t}\right)_{t \in[0, T]}$ define a $\mathcal{E}_{T}$-consistent arbitrage-free emission market, if:

(i) The futures price processes are positively valued.

(ii) There exists a strong ${ }^{4}$ equivalent martingale measure $\mathbb{Q}$, such that the futures price processes are martingales under $\mathbb{Q}$.

(iii) The price processes fulfill the consistency relation:

$$
A_{T}=A_{T}^{\prime}+\pi \mathbf{1}_{\left\{\mathcal{E}_{T} \geq \Lambda+\Gamma\right\}}+\min \left[\left(C_{T}-A_{T}^{\prime}\right)^{+}, \pi\right] \mathbf{1}_{\left\{\Lambda<\mathcal{E}_{T}<\Lambda+\Gamma\right\}}
$$

Moreover, we say that the emission market model fits any reasonable futures curve if for all $\left(\tilde{A}_{0}, \tilde{A}_{0}^{\prime}, \tilde{C}_{0}\right)$, with $\tilde{A}_{0}^{\prime}>0, \tilde{C}_{0}>0$ and $\tilde{A}_{0} \in\left(\tilde{A}_{0}^{\prime}, \tilde{A}_{0}^{\prime}+\pi\right)$ there exist parameters such that $\left(A_{0}, A_{0}^{\prime}, C_{0}\right)=\left(\tilde{A}_{0}, \tilde{A}_{0}^{\prime}, \tilde{C}_{0}\right)$.

This Definition identifies minimal properties that an emission market model for any cap-andtrade schemes similar to EU ETS should fulfill. A straight-forward way to set up such an emission market model is to follow the construction in Corollary 3, but with the constants $a^{\prime}, c, b$ replaced with random variables. This is expressed in the following straight forward Proposition.

Proposition 4. Let $b_{T}$ be defined as $\Lambda+\Gamma-\mathcal{E}_{T}$. Then it holds, that:

(i) For any $a_{T}^{\prime} \in L^{1}\left(\mathcal{F}_{T}\right), c_{T} \in L^{1}\left(\mathcal{F}_{T}\right)$, the processes

$$
\begin{aligned}
A_{t}^{\prime} & =\mathbb{E}_{\mathbb{Q}}\left[a_{T}^{\prime} \exp \left(p X^{*}\right) \mid \mathcal{F}_{t}\right] \\
C_{t} & =\mathbb{E}_{\mathbb{Q}}\left[c_{T} \exp \left(-q X^{*}\right) \mid \mathcal{F}_{t}\right] \\
A_{t} & =A_{t}^{\prime}+\mathbb{E}_{\mathbb{Q}}\left[\pi \mathbf{1}_{\left\{b_{T}<0\right\}}+\max \left[\left(C_{T}-A_{T}^{\prime}\right)^{+}, \pi\right] \mathbf{1}_{\left\{0 \leq b_{T} \leq \Gamma\right\}} \mid \mathcal{F}_{t}\right]
\end{aligned}
$$

with

$$
X^{*}=\max \left[\max \left[\Gamma \wedge m^{*}\left(a_{T}^{\prime}, c_{T}\right), 0\right], \min \left[\Gamma, b_{T}, \frac{\ln \left(c_{T} / a_{T}^{\prime}\right)}{p+q}\right]\right]
$$

are a $\mathcal{E}_{T}$-consistent arbitrage-free emission market.

(ii) Any $\mathcal{E}_{T}$-consistent arbitrage free emission market can be constructed by the procedure defined in (i) using some $a_{T}^{\prime} \in L^{1}\left(\mathcal{F}_{T}\right)$ and $c_{T} \in L^{1}\left(\mathcal{F}_{T}\right)$.

By virtue of the construction of $\left(A_{t}^{\prime}\right)_{t \in[0, T]}$ and $\left(C_{t}\right)_{t \in[0, T]}$, the workings of which appear more involved than a simpler direct specification, a complex interdependence structure can be obtained between $\left(A_{t}^{\prime}\right)_{t \in[0, T]},\left(C_{t}\right)_{t \in[0, T]}$ and $\left(A_{t}\right)_{t \in[0, T]}$, which is linked to the compliance regulations via the equilibrium outcome from Proposition 3, for a simple choice of $a_{T}^{\prime}$ and $c_{T}$. Moreover, depending on specific market structures, this framework leads to realistic price scenarios, demonstrated in Subsection 4.3. It is shown that the price processes $\left(A_{t}^{\prime}\right)_{t \in[0, T]}$ and $\left(C_{t}\right)_{t \in[0, T]}$ are such that prices coincide with positive probability, which as underlined in Proposition 2 (iii), and is a key property of any emission market with a non-zero import limit.

\footnotetext{
${ }^{4}$ This rather strong condition is needed for the pricing of derivatives as we will see in Section 5 .
} 


\subsection{Lognormally driven emission market model}

Greater refinement of the properties of such a model, in particular, whether it is complete, or, whether it can be calibrated to market data, depends on the specific choice of the random variables $a_{T}^{\prime}, c_{T}$ and $b_{T}$. We propose a particular choice that allows the model to be tractable and easy to deal with, but, at the same time, leading to realistic price scenarios.

More precisely, the random variables $a_{T}^{\prime}$ and $c_{T}$ will be considered to be lognormally distributed, and $b_{T}$ will be considered to be a Gaussian random variable. This last assumption is not very strong, as $b_{T}$ is an affine transformation of the market's overall emission, and, therefore, could be similar to a sum of iid random variables.

We now consider a Brownian framework, where the filtration $\mathbb{F}$ is the natural filtration of three one-dimensional Brownian Motions $W^{a}=\left(W_{t}^{a}\right)_{t \in[0, T]}, W^{b}=\left(W_{t}^{b}\right)_{t \in[0, T]}$ and $W^{c}=$ $\left(W_{t}^{c}\right)_{t \in[0, T]}$. These three Brownian motions are correlated, and their correlation matrix $\Pi$ is considered to be constant.

In this refined framework, a lognormally driven emission market model can be defined as follows:

Definition 3. Let the random variables $a_{T}^{\prime}, c_{T}$ and $b_{T}$ derive the value, at time $T$, of the following stochastic processes:

$$
a_{t}^{\prime}=a_{0} \exp \left(\sigma_{a} W_{t}^{a}-\frac{1}{2} \sigma_{a}^{2} t\right) \quad c_{t}=c_{0} \exp \left(\sigma_{c} W_{t}^{c}-\frac{1}{2} \sigma_{c}^{2} t\right) \quad b_{t}=b_{0}+\sigma_{b} W_{t}^{b} .
$$

Thus, the emission $\mathcal{E}_{T}=\Lambda+\Gamma-b_{T}$, together with price processes $\left(A_{t}\right)_{t \in[0, T]},\left(A_{t}^{\prime}\right)_{t \in[0, T]}$ and $\left(C_{t}\right)_{t \in[0, T]}$ defined by (17) - (20), are called a lognormally driven emission market model.

It is obvious from Proposition 4 that this defines a $\mathcal{E}_{T}$-consistent arbitrage-free emission market model. The following result is essential if there is to be a well determined model, as it ensures that there will be a one-to-one correspondence between the market futures prices $\left(A_{t}\right)_{t \in[0, T]}$, $\left(A_{t}^{\prime}\right)_{t \in[0, T]},\left(C_{t}\right)_{t \in[0, T]}$ and the driving processes $\left(a_{t}^{\prime}\right)_{t \in[0, T]},\left(c_{t}\right)_{t \in[0, T]},\left(b_{t}\right)_{t \in[0, T]}$, almost surely.

Proposition 5. For any $0 \leq t \leq T$ and $A_{t}^{\prime *}>0, C_{t}^{*}>0$ and $A_{t}^{*} \in\left(A_{t}^{\prime *}, A_{t}^{\prime *}+\pi\right)$, a unique triple $\left(a_{t}^{\prime}, c_{t}, b_{t}\right)$ with $a_{t}^{\prime} \in\left[A_{t}^{\prime *} \exp (-p \Gamma), A_{t}^{* *}\right]$ and $c_{t} \in\left[C_{t}^{*}, C_{t}^{*} \exp (q \Gamma)\right]$ exists, almost surely, such that the triple $\left(A_{t}^{\prime}, C_{t}, A_{t}\right)$ from Definition 3 matches $\left(A_{t}^{* *}, C_{t}^{*}, A_{t}^{*}\right)$.

Moreover, this proposition highlights the fact that the lognormally driven emission market model can be fitted to any initial term structure with $A_{0}^{\prime *}>0, C_{0}^{*}>0$ and $A_{0}^{*} \in\left(A_{0}^{\prime *}, A_{0}^{\prime *}+\right.$ $\pi)$. It also provides boundaries for the range of the initial parameters, which is crucial for calibration purposes.

\subsection{Interdependence Structures - Some simulation results}

Although the theoretical results are carried out for the general correlation structure, a simpler correlation structure is now used. Namely, we assume that only $W^{a}$ and $W^{b}$ are correlated by a constant $\rho_{a, b}$, meaning that $b_{T}$ and $c_{T}$, as well as $a_{T}^{\prime}$ and $c_{T}$, are independent. This simplifying 
assumption produces more enlightened results, since all correlations between CERs, Phase II and Phase III EUA prices come from the compliance regulation itself.

In this subsection, we will justify our equilibrium based construction of the emission market by showing that this generates a wide range of different interdependence structures, which we anticipate to be typical for different market situations.
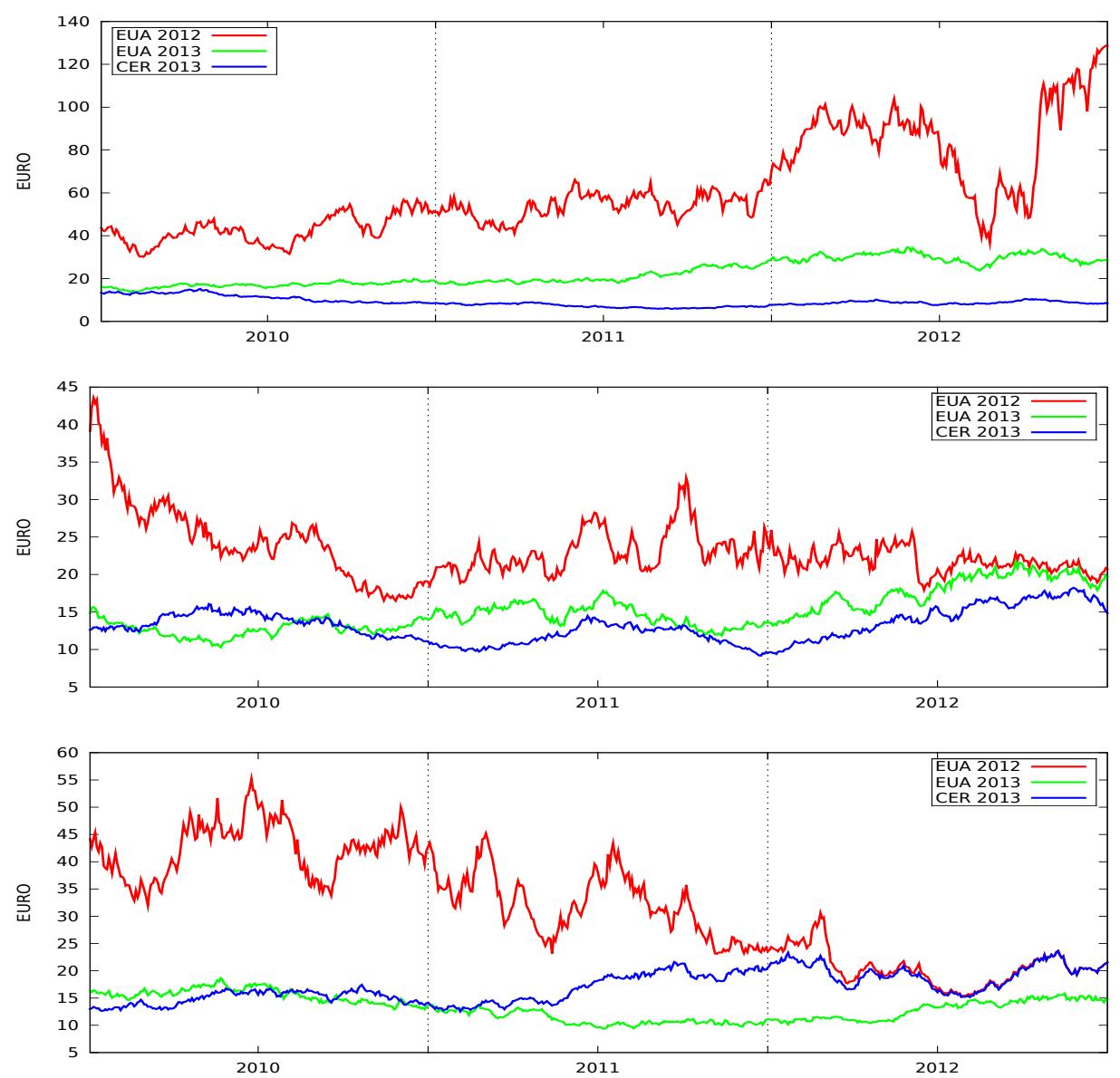

Figure 2: Three simulations of the ongoing period's allowance price process and next period's allowance and CER price processes for the situation where the market is near noncompliance.

Of particular interest are the parameters $b_{0}$ and $\Gamma$, as they determine the compliance situation of the market, which in turn governs the correlation structure of our benchmark futures. As previously explained, $b_{T}$ does not only determine whether the market can cover its emissions or not; if, $b_{T} \wedge \Gamma$ is positive, it describes the width of the compliance interval, i.e. the range by which the number of CERs surrendered for compliance can be altered (in compliance), in order to optimize the number of CERs and Phase III EUAs carried over to the next compliance period. As previously discussed, if $\Gamma-o^{*}$ falls into this compliance interval, the Phase III EUA and CER prices glue together. Hence, increasing the expected value, $b_{0}$, leads to an increase in the probability that CERs and Phase III EUAs will glue together at the end of the period, and consequently, also to an increase of their correlation during the compliance period. 

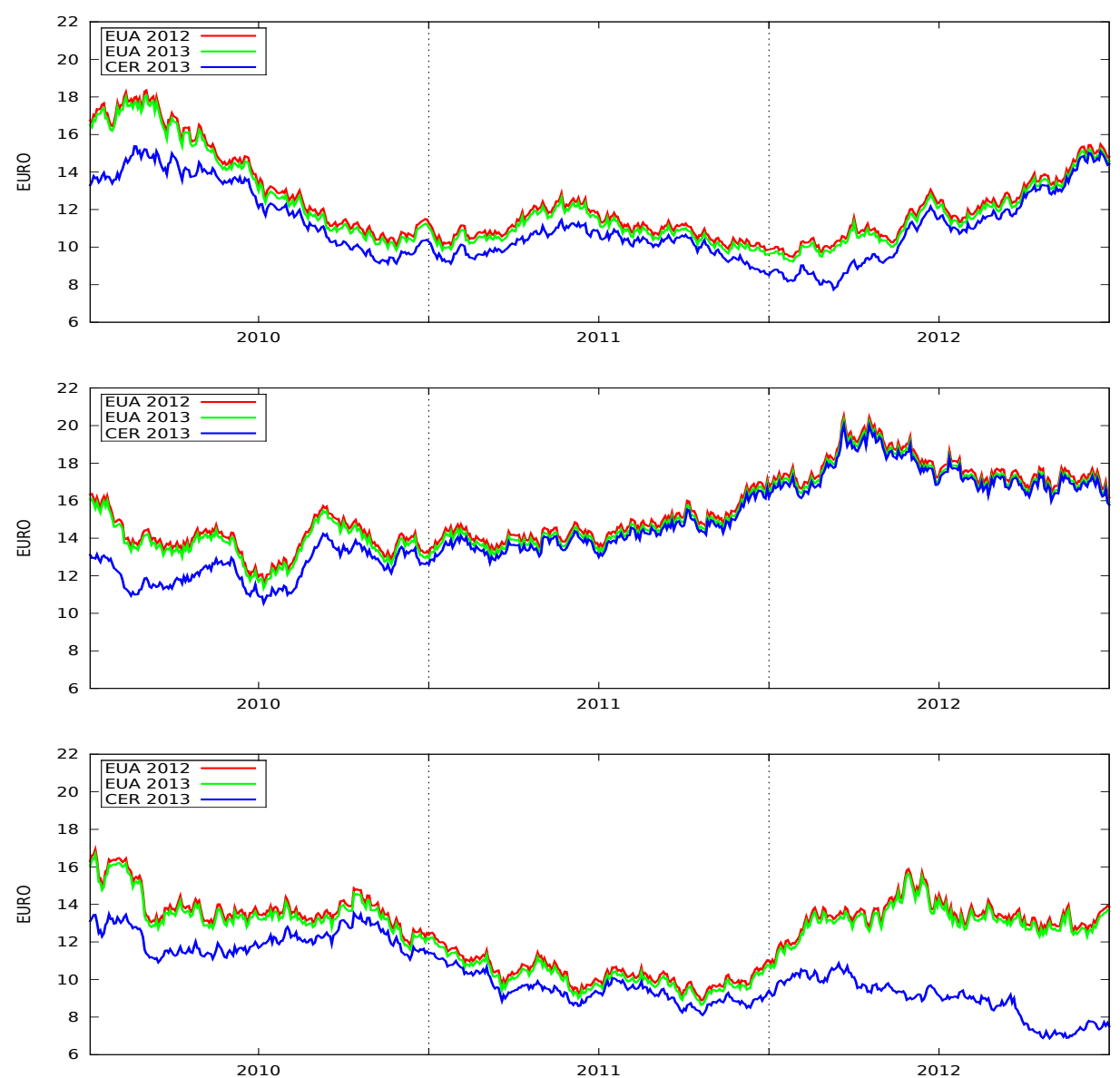

Figure 3: Three simulations of the ongoing period's allowance price process and next period's allowance and CER price processes for the situation where the market is far away from noncompliance.

In the following, we will consider four different compliance situations with different parameters $b_{0}$ and $\Gamma$.

To this end, let $\sigma_{a}=0.34, \sigma_{c}=0.32, p=0.83, q=1.24$ and vary $b_{0}$, as well as $\Gamma$, while we calibrate $a_{0}^{\prime} c_{0}$, to fit the next periods allowance price; and the CER price being 16 and 13 Euros respectively, as of the beginning of April, 2010.

Near a non compliance situation Let us first consider the situation: $b_{0}=0.1 G t, \Gamma=$ $1.4 G t$. Since $b_{0}$ is relatively small, there is a significant probability for noncompliance to take place. In this case, the market needs a lot of CERs for compliance, and $b_{T} \wedge \Gamma$ is relatively small, resulting in a weak correlation between allowances and CERs. This is exactly what we can observe in Figure 2. But, it is not necessary that the market ends in a noncompliance situation. As shown in Figure 2, the situation can also occur that the ongoing period's allowance price ends at the next period's allowance price, or CER price, respectively, depending on which one 

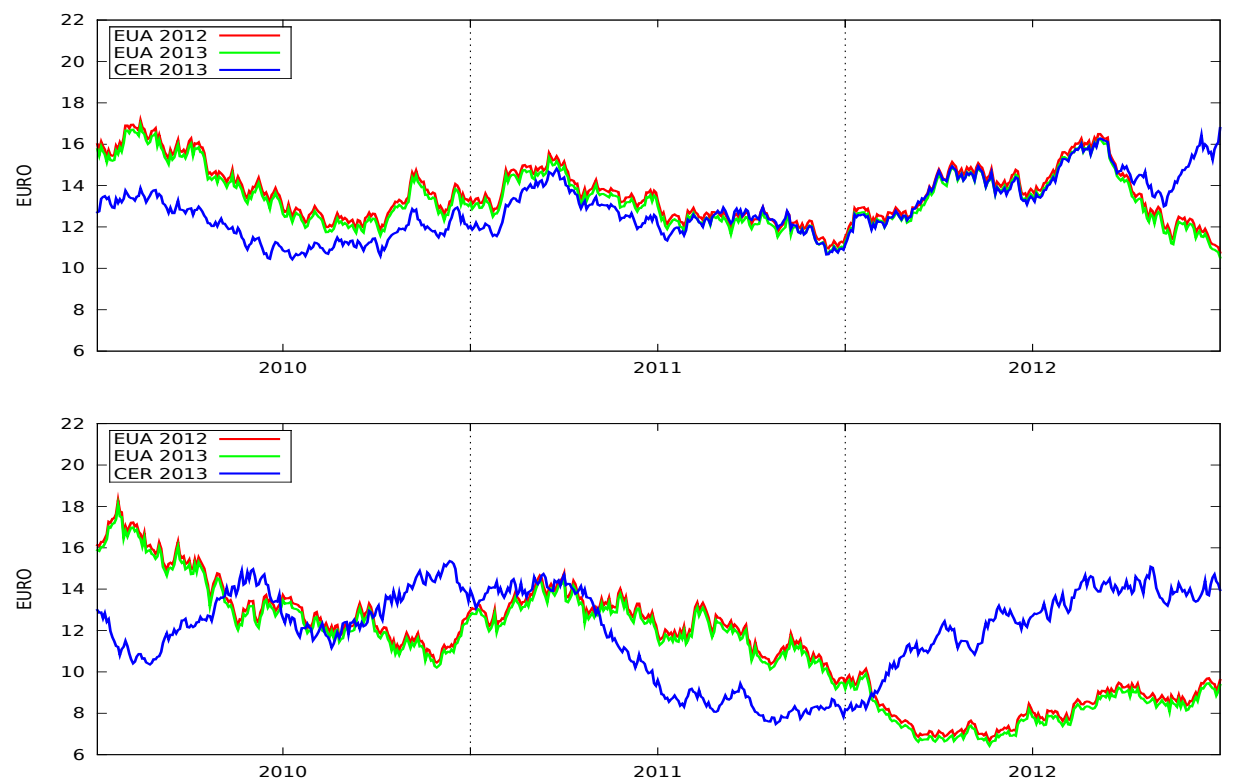

Figure 4: The first pane depicts a simulation, where the EUA price drops below the CER price, because the total emissions can be covered by allowances and no CERs are needed for compliance. The second pane shows a typical simulation, of the case, where no CERs are allowed to be used for compliance.

is greater. In any case, both are a minimum for this period's allowance price. But next period's CER price is not bounded from above by the next period's allowance price.

Today's Situation Today, in the EU ETS, the emissions are not much larger than the initial allocation. Hence, it is very unlikely that we will end up in a noncompliance situation. In line with the calibration (see Appendix), we keep $\Gamma=1.4 G t$, and increase $b_{0}$ to $b_{0}=1.1 G t$. Three simulations for this pair of parameters are displayed in Figure 3. Here, the width $b_{T} \wedge \Gamma$ of the compliance interval is relatively large, leading to a high probability that prices glue together, and, hence, to a significant correlation between the futures. In the first and second simulation, they glue together, once earlier and once later. Another typical situation is that Phase III EUA and CER prices do not glue together, as displayed in the last pane.

Compliance without the use of CERs If the market does not use any CERs for compliance, because the emissions can be covered with the allocated amount of allowances, i.e. $b_{T}>\Gamma$, then the CER price no longer needs to be a lower bound. To bring the market into such a situation, we reduce $\Gamma$ to $\Gamma=0.8$. A typical simulation is depicted in Figure 4. Already, in mid 2011, the CER price exceeds the allowance price slightly. For a couple of month, both stay very correlated, because it takes a while for the CER price to pass the resistance level of the Phase III EUA. Finally the CER price breaks through this resistance level. And, because all emissions can be covered with allowances, it follows that this period's allowance price falls below the CER price and couples to next period's allowance price. 
Small Import Limit From above, it is clear that reducing the import limit, reduces the correlation between EUAs and CERs until it totally disappears for $\Gamma=0$. A simulation for such a situation with $b_{0}=1.1$ is depicted in the lower pane of Figure 4. As expected, both allowance prices are totally independent of the CER price. Moreover, in this case, Phase II EUA and Phase III EUA prices stick together, because $b_{T} \gg 0$; which means that the probability for noncompliance is negligible.

\subsection{Reduced Modeling Framework}

The main objective of the paper is to develop a tractable model that can be implemented easily and is numerically efficient. For this purpose, closed form formulae for the various prices given in Proposition 4, as conditional expected values, are obtained in Appendix E. In this subsection, we go further. More precisely, the paper gives a very general modeling framework, and toolbox, that, when applied to a specific market can be broken down into a simpler model, with less parameters, so that it is easier to handle and calibrate. While our model covers a broad range of different compliance situations, in real world markets, some of these situations may be neglected, in the case that they are considered unlikely to occur. This is, for example, the case in Phase II of the EU ETS. And, indeed, in this subsection we show how to reduce the model to cover only the main aspects of this scheme. As explained above, it is expected that compliance will always take place, and that the Phase III allowance price in the EU ETS is an upper bound for the CER price; meaning that the probability of the event $\left\{A_{T}^{\prime}<C_{T}\right\}$ vanishes, which can be obtained in the limits $p \rightarrow \infty$ and $\Lambda \rightarrow \infty$. In this situation the price processes reduce to

$$
\begin{aligned}
& A_{t} \approx \mathbb{E}\left[a_{T}^{\prime} \mathbf{1}_{\left\{c_{T}<a_{T}^{\prime}\right\}}+\left(a_{T}^{\prime}\right)^{\frac{q}{p+q}}\left(c_{T}\right)^{\frac{p}{p+q}} \mathbf{1}_{\left\{c_{T} \geq a_{T}^{\prime}\right\}} \mid \mathcal{F}_{t}\right] \\
& C_{t} \approx \mathbb{E}\left[c_{T} \mathbf{1}_{\left\{c_{T}<a_{T}^{\prime}\right\}}+\left(a_{T}^{\prime}\right)^{\frac{q}{p+q}}\left(c_{T}\right)^{\frac{p}{p+q}} \mathbf{1}_{\left\{c_{T} \geq a_{T}^{\prime}\right\}} \mid \mathcal{F}_{t}\right]
\end{aligned}
$$

and $A_{t} \approx A_{t}^{\prime}$. This leaves us with only five remaining parameters $\sigma_{a}, \sigma_{c}, p, q, \rho_{a, c}$, where $\rho_{a, c}$ is the correlation between $W^{a}$ and $W^{c}$. This model is used in the Appendix when calibrating these five parameters to the EU ETS.

\section{$5 \quad$ Spread Option Pricing}

In this section, we consider a spread option that allows its owner to exchange one CER for one Phase II EUA. We first prove that the market is complete and show that the option price can be expressed in terms of an adjusted version of Margrabe's formula, when the situation of noncompliance is unlikely to take place. Moreover, we analyze the sensitivity of this option price on some key parameters, which control the EUA and CER price response.

\subsection{Some preliminary comments about spread options}

The spread option is a particularly interesting contract, because it can be hedged via the compliance regulation itself. Indeed, this is done by reserving the corresponding amount of the 
import limit. If the option is exercised, the agent holding a short position, in such an option, can, according to the compliance regulation, exchange a CER for an EUA. On the other hand, if an agent buys such a spread option, the agent's import limit is synthetically increased.

This is an important mechanism, because regulators seem to distribute the import limit equally to the agents in the market, whilst not all agents have the same capabilities to exploit its full option value. Since the option value is typically higher than its intrinsic value (even if it is not the case in the EU ETS today, because of the upper bound property, as seen in Section 2), small agents, without a trading department, can be better off selling their import limit via spread options to companies who have the capability to exploit the full value of the option, by optimally timing the purchase/sale of the CER/EUA or by running an arbitrage strategy against the option.

\subsection{Market completeness and option pricing}

The emission market we consider is arbitrage-free by construction. However, in order to obtain a unique price for the spread option, we first prove the completeness of the market:

Proposition 6. The lognormally driven emission market model, as defined in Definition 3, is complete.

Therefore, applying the standard results of mathematical finance, the price at any time $0 \leq$ $t \leq T$, of a spread option with payoff $\left(A_{T}-C_{T}\right)^{+}$, is uniquely determined as

$$
B_{t} \mathbb{E}\left[\frac{\left(A_{T}-C_{T}\right)^{+}}{B_{T}} \mid \mathcal{F}_{t}\right] .
$$

where $\left(B_{t}\right)_{t \in[0, T]}$ is the numeraire asset, typically the risk-free bank account.

To obtain an explicit closed form formula for the price, an approximation is made, which requires the following assumption.

Assumption 2. The noncompliance event occurs with vanishing probability.

This assumption is not very strong, because regulators seem to calibrate the regulatory parameters such that noncompliance is unlikely to take place. For instance, in the EU ETS, the expected emission is far below the initial allocation plus the import limit of CERs, meaning that noncompliance is nearly impossible. As a consequence we obtain:

Proposition 7. Let the risk-free rate $r$ be constant. Then, in the lognormally driven emission market model, under Assumption 2, the spread option price can be approximated by:

$$
B_{t} \mathbb{E}\left[\frac{\left(A_{T}^{\prime}-C_{T}\right)^{+}}{B_{T}} \mid \mathcal{F}_{t}\right]=B_{t} \mathbb{E}\left[\frac{\left(a_{T}^{\prime}-c_{T}\right)^{+}}{B_{T}} \mid \mathcal{F}_{t}\right]=e^{-r(T-t)}\left(a_{T}^{\prime} \Phi\left(d_{a}\right)-c_{T}^{\prime} \Phi\left(d_{c}\right)\right)
$$

with

$$
d_{a}=\frac{\ln \left(a_{t}^{\prime} / c_{t}^{\prime}\right)+\frac{1}{2} \sigma^{2} T}{\sigma \sqrt{T}} \quad d_{c}=\frac{\ln \left(a_{t}^{\prime} / c_{t}^{\prime}\right)-\frac{1}{2} \sigma^{2} T}{\sigma \sqrt{T}} \quad \sigma^{2}=\sigma_{a}^{2}+\sigma_{c}^{2}-2 \rho_{a, c} \sigma_{a} \sigma_{c} .
$$



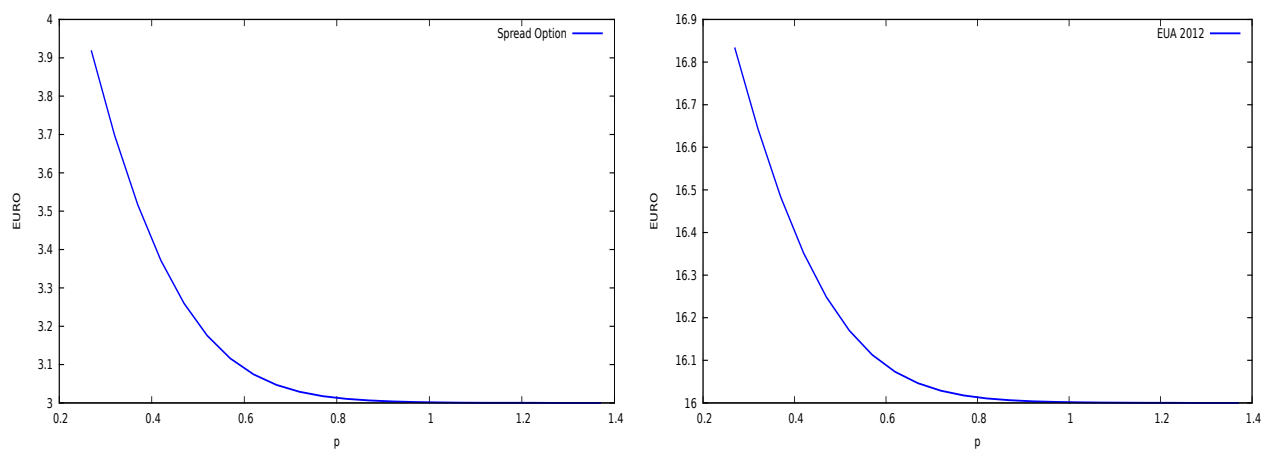

Figure 5: Dependence of spread option price and Phase II EUA price on the price response parameter.

Note that this formula can be extended to the framework of deterministic, or stochastic, interest rates.

Note also that the standard black scholes setting, where $\left(A_{t}^{\prime}\right)_{t \in[0, T]}$, and $\left(C_{t}\right)_{t \in[0, T]}$, follow geometric Brownian motions, leading to Margrabe's Formula for the spread option price. This is a particular limit case of the formula given in Proposition 7 and corresponds to the case where the price response parameter, $p$, is equal to zero. In the next subsection, we look at the sensitivity of the spread option price to the parameter $p$, and highlight the importance of modeling the price response.

\subsection{Calibration and sensitivity analysis}

Following the calibration procedure, described in Appendix D, we calibrate the emission market model implicitly to the futures curve observed on 24/8/2010 in the EU ETS. We are now interested in pricing and studying the sensitivity of a spread option with a maturity of $31 / 12 / 2012$.

As shown in Figure 5, it is unsurprising that the price response parameter can have a significant impact upon the price of the spread option, since it represents whether the next period's allowance price is an upper bound for the CER price, or not. For a large price response, the price of the spread option is given by its intrinsic value $A_{0}^{\prime}-C_{0}$, as the next period EUA price is an upper bound for the CER price. On the other hand, for a small price response, where both next period's allowance and the CER price behave like geometric Brownian motions, this is no longer the case: the price of the spread option corresponds to the Margrabe's formula, but applied to $A_{0}^{\prime}$ and $C_{0}$.

While the Phase III EUA price and the CER price are obtained through the calibration, the ongoing period's EUA price depends, significantly, on the price response. As can be seen from Equations (27)-(29), Phase II and Phase III EUA prices at time $T$ differ only in two situations. Firstly, on the set $N$, which occurs with vanishing probability. And, secondly, on the set $F$, where $\Lambda<\mathcal{E}_{T} \leq \Lambda+\Gamma$ and $C_{T}>A_{T}^{\prime}$, which implies that the Phase II EUA price equals the CER price. 
Hence, the ongoing and next period's CER prices converge if the price response is strong, and the CER price is bounded from above by the Phase III EUA price. On the other hand, if the price response is weak, then the CER price is more likely to exceed the Phase III EUA price, in which case, the Phase II EUA price couples to the CER price. This effect drives a wedge between the Phase II and Phase III EUA price when the price response is weakened, and is what we see in the right pane of Figure 5. Therefore, the difference between the ongoing and next period's allowance price can be interpreted as a good indicator for the price response.

\section{Conclusions}

In this paper, we propose a model for market consistent futures price dynamics for EUA and CER cap-and-trade schemes. Thereby, we discuss the impact of the regulation on the different emission certificates using an equilibrium model. Based on these insights, we propose a general modeling framework for arbitrage-free futures price processes, which are consistent with the regulation with reference to our equilibrium results. We show that this market model is complete, tractable and can be calibrated to market data. The aim was to provide a very general toolbox that can be broken down into simpler models, which cover very specific markets, and we illustrate how this can be done for the EU ETS. Moreover, we analyze simulations of futures price processes, and show that it generates a wide range of different interdependence structures between our benchmark futures, which we anticipate are typical for different market situations. Finally, we calibrate the model parameters to the second Phase of the EU ETS, and apply our framework to the pricing of spread options between EUAs and CERs - which, per se, is a very interesting contract, since it allows the import limit (a key regulatory parameter) to be traded.

\section{A Proof of Partial Equilibrium Results}

To prove Propositions 1 and 2, it is helpful to reformulate the representative planer problem, 5 , as follows

$$
\begin{array}{cl}
\inf _{\gamma, Z} & \pi Z-\mathcal{A}(\Lambda-\mathcal{E}+\gamma)-\mathcal{C}(-\gamma) \\
\text { s.t. } & Z \geq \mathcal{E}-\Lambda-\gamma, \quad Z \geq 0 \\
& 0 \leq \gamma \leq \Gamma
\end{array}
$$

Due to Assumption 1 this is a convex optimization problem, and $\gamma \mapsto \mathcal{C}^{\prime}(-\gamma)-\mathcal{A}^{\prime}(\mathcal{E}-\Lambda+\gamma)$ is strong and monotonically increasing.

Proof of Proposition 1. Problem (22) fulfills Slater's condition, and, hence, its optimal solution $(\bar{\gamma}, \bar{Z})$ is given as a solution to the Karush Kuhn Tucker (KKT) conditions, given as follows. Stationarity conditions:

$$
\mathcal{C}^{\prime}(-\bar{\gamma})-\mathcal{A}^{\prime}(\mathcal{E}-\Lambda+\bar{\gamma})=\mu_{1}-\mu_{3}+\mu_{4}, \quad \pi=\mu_{1}+\mu_{2}
$$


Complementary slackness conditions:

$$
\mu_{1}(\mathcal{E}-\Lambda-\bar{\gamma}-\bar{Z})=0, \quad \mu_{2} \bar{Z}=0, \quad \mu_{3}(\bar{\gamma}-\Gamma)=0, \quad \mu_{4} \bar{\gamma}=0 .
$$

Primal feasibility:

$$
0 \leq \bar{\gamma} \leq \Gamma, \quad \bar{Z} \geq 0, \quad \bar{Z} \geq \mathcal{E}-\Lambda-\bar{\gamma}
$$

Dual feasibility:

$$
\mu_{1} \geq 0, \quad \mu_{2} \geq 0, \quad \mu_{3} \geq 0, \quad \mu_{4} \geq 0, \quad
$$

To prove Proposition 1, it is enough to show that the KKT conditions imply the following: (i) If, $\bar{\gamma}=0$, then $\min \left[m^{*}, \max \left[\mathcal{E}-\Lambda, o^{*}\right]\right] \leq 0$. (ii) If else, $0<\bar{\gamma}<\min \left[m^{*}, \Gamma\right]$, then $\bar{\gamma}=\max \left[\mathcal{E}-\Lambda, o^{*}\right]$. (iii) If, $\bar{\gamma}=\min \left[m^{*}, \Gamma\right]>0$ then $\max \left[\mathcal{E}-\Lambda, o^{*}\right] \geq \min \left[m^{*}, \Gamma\right]$.

Let us first prove (ii) and assume, therefore, that $0<\bar{\gamma}<\min \left[m^{*}, \Gamma\right]$, which implies $\mu_{3}=$ $\mu_{4}=0$. Since $\bar{\gamma}<m^{*}$, it holds that $\mathcal{C}^{\prime}(-\bar{\gamma})<\mathcal{A}^{\prime}(\mathcal{E}-\Lambda+\bar{\gamma})+\pi$, and, hence, $0 \leq \mu_{1}<\pi$. We have to distinguish two cases. First, $\mu_{1}=0$. Then it holds by definition that $\bar{\gamma}=o^{*}$. Since, moreover, $\mu_{1}=0$, it follows that $\mu_{2}=\pi$, and, hence, $\bar{Z}=0$ and $\mathcal{E}-\Lambda \leq \bar{\gamma}$. Hence, in this case (ii) holds true. Secondly, if, $0<\mu_{1}<\pi$, then $\mu_{2}>0$, and consequently $\bar{Z}=0$, as well as $\mathcal{E}-\Lambda=\bar{\gamma}$. Since $\mathcal{C}(-\bar{\gamma})-A^{\prime}(\mathcal{E}-\Lambda+\bar{\gamma})=\mu_{1}>0$, it also follows that $\bar{\gamma}>o^{*}$. And again (ii) holds true.

Now, let us turn to (iii), and suppose that $\bar{\gamma}=\min \left[m^{*}, \Gamma\right]>0$, which implies that $\mu_{4}=0$. It is obvious that (iii) holds, if, $o^{*} \geq \min \left[m^{*}, \Gamma\right]$. If, on the other hand, $o^{*}<\min \left[m^{*}, \Gamma\right]=\bar{\gamma}$, then it holds that $\mathcal{C}^{\prime}(-\bar{\gamma})-\mathcal{A}^{\prime}(\mathcal{E}-\Lambda+\bar{\gamma})>0$, and consequently $\mu_{1}>0$ and $\mathcal{E}-\Lambda-\bar{\gamma}=\bar{Z} \geq 0$, which implies that $\mathcal{E}-\Lambda \geq \bar{\gamma}$, and, hence (iii) holds true.

Now, consider assertion (i). We assume $\bar{\gamma}=0$, and notice that this implies $\mu_{3}=0$, and, hence, $\mathcal{C}^{\prime}(0)-\mathcal{A}^{\prime}(\mathcal{E}-\Lambda)=\mu_{1}+\mu_{4} \geq 0$, which implies that $o^{*} \leq 0$. The assertion is trivially fulfilled if $m^{*} \leq 0$. If, on the other hand, $m^{*}>0$, then it holds that $\mathcal{C}^{\prime}(0)-\mathcal{A}^{\prime}(\mathcal{E}-\Lambda)<\pi$, and, hence, $\mu_{1}<\pi$, as well as $\mu_{2}>0$, which implies that $\bar{Z}=0$. Due to primal feasibility $\bar{Z} \geq \mathcal{E}-\Lambda$, this proves that $0 \geq \mathcal{E}-\Lambda$, which concludes the assertion.

To derive the equilibrium prices, the following Lemma is shown to be helpful. It is a simple consequence of Assumption 1.

Lemma 1. Let $\eta \in \mathbb{R}^{|I|}$ and $\beta \in \mathbb{R}^{|J|}$, such that $\mathcal{A}^{i^{\prime}}\left(\eta^{i}\right)=\mathcal{A}^{k^{\prime}}\left(\eta^{k}\right)$ for all $i, k \in I$ and $\mathcal{C}^{j^{\prime}}\left(\beta^{j}\right)=\mathcal{C}^{l^{\prime}}\left(\beta^{l}\right)$ for all $j, l \in J$. Then it holds that $\mathcal{A}^{\prime}\left(\sum_{i \in I} \eta^{i}\right)=\mathcal{A}^{i^{\prime}}\left(\eta^{i}\right)$ for all $i \in I$, as well as $\mathcal{C}^{\prime}\left(\sum_{j \in J} \beta^{j}\right)=\mathcal{C}^{j^{\prime}}\left(\beta^{j}\right)$, for all $J \in J$.

The following proof will involve the aggregated planer problem, given by:

$$
\begin{array}{ll}
\inf & {\left[\sum_{i \in I} \pi Z^{i}-\mathcal{A}^{i}\left(-\mathcal{E}^{i}+\Lambda^{i}+\phi^{i}+\varphi^{i}+\gamma^{i}\right)\right]-\sum_{j \in J} \mathcal{C}^{j}\left(\beta^{j}\right)} \\
\text { s.t. } & \sum_{i \in I} \phi^{i}=0, \quad \sum_{i \in I} \varphi^{i}=0, \quad \sum_{i \in I} \gamma^{i}=\sum_{j \in J} \beta^{j} \\
& Z^{i} \geq \mathcal{E}^{i}-\Lambda^{i}-\phi^{i}-\gamma^{i}, \quad Z^{i} \geq 0, \quad 0 \leq \gamma^{i} \leq \Gamma^{i}, \quad i \in I .
\end{array}
$$

It optimizes the aggregated projected P\&L functions under all individual constraints, as well as the trading constraints. This is interesting, since we obtain the dual variables of the trading 
constraints, which correspond to equilibrium prices. This is in contrast to the representative planer problem (5), where not only the projected P\&L functions are aggregated, but the compliance strategies are also reduced to a single real number, namely the amount of CERs surrendered by the overall market.

Proof of Proposition 2. (i) Due to Assumption 1, Problem (23) fulfills the Slater conditions. Hence, it has optimal primal and dual solutions $(\bar{\phi}, \bar{\varphi}, \bar{\gamma}, \bar{\beta}, \bar{Z})$ and $\left(\bar{A}, \bar{A}^{\prime}, \bar{C}\right)$, respectively, and strong duality, holds. If we denote the optimal value of (23) by $P^{*}$, then weak duality implies

$$
\begin{aligned}
& \sup _{\left(A, A^{\prime}, C\right) \in \mathbb{R}_{+}^{3}}\left[\sum_{i \in I} \inf _{\left(\phi^{i}, \varphi^{i}, \gamma^{i}\right) \in \mathcal{S}^{i}} L^{A, A^{\prime}, C, i}\left(\phi^{i}, \varphi^{i}, \gamma^{i}\right)+\sum_{j \in J} \inf _{\beta^{j} \in \mathcal{S}^{j}} M^{C, i}\left(\beta^{i}\right)\right] \\
& =\sum_{i \in I} \inf _{\left(\phi^{i}, \varphi^{i}, \gamma^{i}\right) \in \mathcal{S}^{i}} L^{\bar{A}, \bar{A}^{\prime}, \bar{C}, i}\left(\phi^{i}, \varphi^{i}, \gamma^{i}\right)+\sum_{j \in J} \inf _{\beta^{j} \in \mathcal{S}^{j}} M^{\bar{C}, i}\left(\beta^{i}\right) \\
& \leq \sum_{i \in I} L^{\bar{A}, \bar{A}^{\prime}, \bar{C}, i}\left(\bar{\phi}^{i}, \bar{\varphi}^{i}, \bar{\gamma}^{i}\right)+\sum_{j \in J} M^{\bar{C}, j}\left(\bar{\beta}^{j}\right)=P^{*} .
\end{aligned}
$$

Due to strong duality, the inequality holds with equality, and we conclude that $L^{\bar{A}, \bar{A}^{\prime}, \bar{C}, i}\left(\bar{\phi}^{i}, \bar{\varphi}^{i}, \bar{\gamma}^{i}\right) \leq L^{\bar{A}^{\bar{A}}, \bar{A}^{\prime}, \bar{C}, i}\left(\phi^{i}, \varphi^{i}, \gamma^{i}\right)$ for all $\left(\phi^{i}, \varphi^{i}, \gamma^{i}\right) \in \mathcal{S}^{i}, i \in I$ as well as $M^{\bar{C}, j}\left(\bar{\beta}^{j}\right) \leq$ $M^{\bar{C}, j}\left(\beta^{j}\right)$ for all $\beta^{j} \in \mathcal{S}^{j}, j \in J$. This proves that the triple $\left(\bar{A}, \bar{A}^{\prime}, \bar{C}\right)$ form an equilibrium with strategies $(\bar{\phi}, \bar{\varphi}, \bar{\gamma}, \bar{\beta})$.

(ii) Now let us derive equilibrium prices, and show that they can be expressed in terms of the optimal solution of the representative planer problem (5). To this end, we specify the Lagrange multipliers, analyzing the Kuhn Karush Tucker conditions of the aggregated planer problem (23).

The stationarity conditions read:

$$
\begin{aligned}
\bar{A} & =\mathcal{A}^{i}\left(-\mathcal{E}^{i}+\Lambda^{i}+\bar{\phi}^{i}+\bar{\varphi}^{i}+\bar{\gamma}^{i}\right)+\mu_{1}^{i} & & \pi=\mu_{1}^{i}+\mu_{2}^{i} \\
\bar{A}^{\prime} & =\mathcal{A}^{i}\left(-\mathcal{E}^{i}+\Lambda^{i}+\bar{\phi}^{i}+\bar{\varphi}^{i}+\bar{\gamma}^{i}\right) & & \bar{C}=\mathcal{C}^{j}\left(\bar{\beta}^{j}\right) \\
\bar{C}-\bar{A}^{\prime} & =\mu_{1}^{i}-\mu_{3}^{i}+\mu_{4}^{i} & &
\end{aligned}
$$

Lemma 1 together with (25) prove (8) and (9). Furthermore, from (24) we conclude that $\mu_{1}^{i}=\mu_{1}^{i^{\prime}}$ and $\mu_{2}^{i}=\mu_{2}^{i^{\prime}}$ for all $i, i^{\prime} \in I$. Moreover the complementary slackness conditions are given by

$$
\mu_{1}^{i}\left(\mathcal{E}^{i}-\Lambda^{i}-\bar{\phi}^{i}-\bar{\gamma}^{i}-\bar{Z}^{i}\right)=0, \quad \mu_{2}^{i} \bar{Z}^{i}=0, \quad \mu_{3}^{i}\left(\bar{\gamma}^{i}-\Gamma^{i}\right)=0, \quad \mu_{4}^{i} \bar{\gamma}^{i}=0
$$

It is easy to see that (26), together with the third and fourth complementary slackness conditions, imply that $\mu_{3}^{i}=\left(A^{\prime}-C+\mu_{1}^{i}\right)^{+}$as well as $\mu_{4}^{i}=\left(C-A^{\prime}-\mu_{1}\right)^{+}$for all $i \in I$. Consequently it holds that $\mu_{3}^{i}=\mu_{3}^{i^{\prime}}$ and $\mu_{4}^{i}=\mu_{4}^{i^{\prime}}$ for all $i, i^{\prime} \in I$. Since $\mu_{1}=\mu_{1}^{i}, \mu_{2}=\mu_{2}^{i}, \mu_{3}=\mu_{3}^{i}$ and $\mu_{4}=\mu_{4}^{i}$ for all $i \in I$ it follows that our KKT conditions reduce to the KKT conditions from Proposition 1 with aggregated quantities. Hence, $\sum_{i \in I} \overline{\gamma^{i}}$ coincides with the optimal solution of (5).

Now let us prove (10) by identifying necessary conditions for $\mu_{1}$ from the KKT conditions of Proposition 1. If $\mathcal{E}>\Lambda+\Gamma$ it follows that $\bar{Z}=\mathcal{E}-\Lambda-\bar{\gamma}>0$ with $\mu_{2}=0, \mu_{1}=\pi$ and $\bar{A}=\bar{A}^{\prime}+\pi$. If $\mathcal{E}<\Lambda$ it follows that $\mathcal{E}-\Lambda-\bar{\gamma}-\bar{Z}<0$, and, hence, $\mu_{1}=0$ and $\bar{A}=\bar{A}^{\prime}$. 
If, $\Lambda<\mathcal{E}<\Lambda+\Gamma$, we have to distinguish three different situations. First, if, $\bar{C}-\bar{A}^{\prime}>\pi$, it follows that $\mu_{4}>0$, and, hence, $\bar{\gamma}=0$ and $\bar{Z}>0$. This implies that $\mu_{2}=0$ and $\mu_{1}=\pi$. Secondly, if, $\bar{C}-\bar{A}^{\prime}<0$ then $\mu_{3}>0$ and $\bar{\gamma}=\Gamma$. Consequently it holds that $\mathcal{E}-\Lambda-\bar{\gamma}<0$ and $\mu_{1}=0$. The third case is that $0 \leq \bar{C}-\bar{A}^{\prime} \leq \pi$. Here, it follows from the KKT conditions that $\mathcal{E}-\Lambda-\bar{\gamma}=0$. Hence, $0<\bar{\gamma}<\Gamma$ and $\mu_{3}=\mu_{4}=0$, which implies that $\mu_{1}=\bar{C}-\bar{A}$. It remains to identify $\bar{A}$ if $\mathcal{E}=\Lambda+\Gamma$ and $\mathcal{E}=\Lambda$. In both cases, the allowance price is not unique, but it can be shown that $\mu_{1}=\pi$ and $\mu_{1}=0$ respectively posses valid solutions to the KKT conditions.

(iii) This is again a direct consequence of the KKT conditions of the representative agent problem. From $\mathcal{E}-\Lambda-\bar{\gamma}<0$ it follows that $\mu_{1}=0$. Since, moreover, $0<\bar{\gamma}<\Gamma$, we obtain $\mu_{3}=0$, as well as $\mu_{4}=0$, and conclude that $C=A^{\prime}$.

Proof of Proposition 3. Assertion (i) is a direct consequence of Proposition 2. Let us turn to (ii). If $C \leq A^{\prime}$ let $c=C, a^{\prime}=A^{\prime}$. Then $m^{*}\left(a^{\prime}, c\right)<\frac{\ln \left(c / a^{\prime}\right)}{p+q} \leq 0$, and consequently $x^{*}\left(a^{\prime}, c, b\right)=0$, which proves that (15) hold true. Moreover, if $C \geq A^{\prime}+\pi$, let $x=\Gamma$, and define $c, a^{\prime}$ by $c=C \exp (q x)$ and $a^{\prime}=A^{\prime} \exp (-p x)$. Since, $m^{*} \geq \Gamma$, it follows that $x^{*}\left(a^{\prime}, c, b\right)=\Gamma$, which proves (15). If $A^{\prime}<C<A^{\prime}+\pi$, let $x=\max [0, \min [b, \Gamma]]$, and define, again, $c, a^{\prime}$, as above. Then, $\frac{\ln \left(c / a^{\prime}\right)}{p+q}>x>m^{*}\left(a^{\prime}, c\right)$ i.e. $\frac{\ln \left(c / a^{\prime}\right)}{p+q}>0$ and $\frac{\ln \left(c / a^{\prime}\right)}{p+q}>\min [b, \Gamma]$, as well as $m^{*}\left(a^{\prime}, c\right)<0$ or $m^{*}\left(a^{\prime}, c\right)<\min [b, \Gamma]$. Consequently we obtain $x=x^{*}\left(a^{\prime}, c, b\right)$, which proves the assertion.

\section{B Proof of Market Completeness and Spread Option Prices}

To prove market completeness, we start with a straight-forward result that specifies the SDE of $\left(A, A^{\prime}, C\right)$. Without loss of generality, we assume throughout this Section that $p=1$.

To this end, we reformulate the Phase III EUA and CER prices by

$$
\begin{aligned}
& A_{t}^{\prime}=\mathbb{E}\left[a_{T}^{\prime} \mathbf{1}_{\{D\}}+\left(a_{T}^{\prime}\right)^{\frac{q}{1+q}}\left(c_{T}\right)^{\frac{1}{1+q}} \mathbf{1}_{\{E\}}+a_{T}^{\prime} \mathrm{e}^{b_{T}} \mathbf{1}_{\{F\}}+a_{T}^{\prime} \mathrm{e}^{\Gamma} \mathbf{1}_{\{G\}}+a_{T}^{\prime} \mathrm{e}^{m^{*}} \mathbf{1}_{\{H\}} \mid \mathcal{F}_{t}\right] \\
& C_{t}=\mathbb{E}\left[c_{T} \mathbf{1}_{\{D\}}+\left(a_{T}^{\prime}\right)^{\frac{q}{1+q}}\left(c_{T}\right)^{\frac{1}{1+q}} \mathbf{1}_{\{E\}}+c_{T} \mathrm{e}^{-q b_{T}} \mathbf{1}_{\{F\}}+c_{T} \mathrm{e}^{-q \Gamma} \mathbf{1}_{\{G\}}+c_{T} \mathrm{e}^{-q m^{*}} \mathbf{1}_{\{H\}} \mid \mathcal{F}_{t}\right]
\end{aligned}
$$

as well as phase II EUA prices by

$$
A_{t}=\mathbb{E}\left[a_{T}^{\prime} \mathbf{1}_{\{D\}}+\left(a_{T}^{\prime}\right)^{\frac{q}{1+q}}\left(c_{T}\right)^{\frac{1}{1+q}} \mathbf{1}_{\{E\}}+c_{T} \mathrm{e}^{-q b_{T}} \mathbf{1}_{\{F\}}+a_{T}^{\prime} \mathrm{e}^{\Gamma} \mathbf{1}_{\{G\}}+a_{T}^{\prime} \mathrm{e}^{m^{*}} \mathbf{1}_{\{H\}}+\pi \mathbf{1}_{\{N\}} \mid \mathcal{F}_{t}\right] .
$$

For this reformulation, we used the following obvious index sets:

$$
\begin{array}{lr}
D=\left\{\min \left(b_{T}, \ln \left(\frac{c_{T}}{a_{T}^{\prime}}\right)\right)<0\right\} \cap\left\{m^{*} \leq 0\right\} & G=\left\{\min \left(b_{T}, \frac{\ln \left(c_{T} / a_{T}^{\prime}\right)}{1+q}\right) \geq \Gamma\right\} \cup\left\{b_{T}<\kappa<m^{*}\right\} \\
E=\left\{0 \leq \frac{\ln \left(c_{T} / a_{T}^{\prime}\right)}{1+q}<\Gamma\right\} \cap\left\{b_{T}>\frac{\ln \left(c_{T} / a_{T}^{\prime}\right)}{1+q}\right\} & H=\left\{b_{T} \vee 0<m^{*} \leq \Gamma\right\} \\
F=\left\{0 \wedge m^{*} \leq b_{T}<\Gamma\right\} \cap\left\{\frac{\ln \left(c_{T} / a_{T}^{\prime}\right)}{1+q} \geq b_{T}\right\} & N=\left(\left\{b_{T}<0\right\} \cap\left\{m^{*} \leq 0\right\}\right) \cup\left\{m^{*} \wedge \Gamma>b_{T} \vee 0\right\} .
\end{array}
$$

Based on these sets, we also define the following random matrix

$$
\Sigma_{t}=\left(\begin{array}{ccc}
a+b+c+d & b^{\prime} & a_{t}^{\prime} c \\
b & e+b^{\prime}+f+g & -q c_{t} f \\
a+b+d & b^{\prime}+f & z-q c_{t} f
\end{array}\right)
$$


for all $t \in[0, T)$ almost surely, where the matrix elements are given by

$$
\begin{array}{rlr}
a & =\frac{1}{a_{t}^{\prime}} \mathbb{E}\left[a_{T}^{\prime} \mathbf{1}_{\{D\}} \mid \mathcal{F}_{t}\right] & e=\frac{1}{c_{t}} \mathbb{E}\left[c_{T} \mathbf{1}_{\{D\}} \mid \mathcal{F}_{t}\right] \\
c=\frac{1}{a_{t}^{\prime}} \mathbb{E}\left[a_{T}^{\prime} e^{b_{T}} \mathbf{1}_{\{F\}} \mid \mathcal{F}_{t}\right] & f=\frac{1}{c_{t}} \mathbb{E}\left[c_{T} e^{-q b_{T}} \mathbf{1}_{\{F\}} \mid \mathcal{F}_{t}\right] \\
d=\frac{1}{a_{t}^{\prime}} \mathbb{E}\left[a_{T}^{\prime} e^{\Gamma} \mathbf{1}_{\{G\}} \mid \mathcal{F}_{t}\right] & g=\frac{1}{c_{t}} \mathbb{E}\left[c_{T} e^{-q \Gamma} \mathbf{1}_{\{G\}} \mid \mathcal{F}_{t}\right] .
\end{array}
$$

and

$$
\begin{aligned}
b & =\frac{q}{1+q} \frac{1}{a_{t}^{\prime}} \mathbb{E}\left[a_{T}^{\frac{q}{1+q}} c_{T}^{\frac{1}{1+q}} \mathbf{1}_{\{E\}} \mid \mathcal{F}_{t}\right]-\frac{q}{a_{t}^{\prime}} \mathbb{E}\left[a_{T}^{\prime} c_{T} \exp \left(-q m^{*}\left(a_{T}^{\prime}, c_{T}\right)\right) \frac{\partial m^{*}\left(a_{T}^{\prime}, c_{T}\right)}{\partial a^{\prime}} \mathbf{1}_{\{H\}} \mid \mathcal{F}_{t}\right] \\
b^{\prime} & =\frac{1}{1+q} \frac{1}{c_{t}} \mathbb{E}\left[a_{T}^{\frac{q}{1+q}} c_{T}^{\frac{1}{1+q}} \mathbf{1}_{\{E\}} \mid \mathcal{F}_{t}\right]+\frac{1}{c_{t}} \mathbb{E}\left[c_{T} a_{T}^{\prime} \exp \left(m^{*}\left(a_{T}^{\prime}, c_{T}\right)\right) \frac{\partial m^{*}\left(a_{T}^{\prime}, c_{T}\right)}{\partial c} \mathbf{1}_{\{H\}} \mid \mathcal{F}_{t}\right]
\end{aligned}
$$

as well as

$$
z_{t}=\frac{\partial}{\partial x} \mathbb{E}\left[A_{t} \mid b_{t}=x\right]+q c_{t} f .
$$

In the following Lemma we will show that $\Sigma_{t} \Pi$ is the volatility matrix of the price processes.

Lemma 2. For $t \in[0, T)$ the dynamics of $\left(A, A^{\prime}, C\right)$ follow the stochastic differential equation

$$
d\left(\begin{array}{c}
A_{t}^{\prime} \\
C_{t} \\
A_{t}
\end{array}\right)=\Sigma_{t}\left(\begin{array}{c}
d a_{t}^{\prime} \\
d c_{t} \\
d b_{t}
\end{array}\right)=\Sigma_{t} \Pi\left(\begin{array}{c}
d W_{t}^{a} \\
d W_{t}^{c} \\
d W_{t}^{b}
\end{array}\right)
$$

almost surely.

Proof of Lemma 2. Let us exemplarily compute $\partial A_{t}^{\prime} / \partial a_{t}^{\prime}$ and $\partial A_{t}^{\prime} / \partial b_{t}$. To this end, we define $g\left(a^{\prime}, c, b\right)=a^{\prime} \cdot \exp \left(x^{*}\left(a^{\prime}, c, b\right)\right) \quad x^{*}\left(a^{\prime}, c, b\right)=\max \left[\max \left[\Gamma \wedge m^{*}\left(a^{\prime}, c\right), 0\right], \min \left[\Gamma, b, \frac{\ln \left(c / a^{\prime}\right)}{p+q}\right]\right]$

Then, we obtain

$$
\frac{\partial A_{t}^{\prime}}{\partial a_{t}^{\prime}}=\mathbb{E}_{\mathbb{Q}}\left[\left(\frac{\partial a_{T}^{\prime}}{\partial a_{t}^{\prime}}\right) \frac{\partial}{\partial a} g\left(a_{T}^{\prime}, c_{T}, b_{T}\right) \mid \mathcal{F}_{t}\right]=\mathbb{E}_{\mathbb{Q}}\left[\left(\frac{a_{T}^{\prime}}{a_{t}^{\prime}}\right) \frac{\partial}{\partial a} g\left(a_{T}^{\prime}, c_{T}, b_{T}\right) \mid \mathcal{F}_{t}\right]
$$

where we used

$$
\frac{\partial a_{T}^{\prime}}{\partial a_{t}^{\prime}}=\exp \left(\sigma_{a}\left(W_{T}^{a}-W_{t}^{a}\right)-\frac{1}{2} \sigma_{a}^{2}(T-t)\right)=\frac{a_{T}^{\prime}}{a_{t}^{\prime}} .
$$

Since the right and left hand derivatives of $x^{*}$ are given by

$$
\begin{aligned}
& \frac{\partial}{\partial_{+} a^{\prime}} x^{*}\left(a^{\prime}, c, b\right)=-\frac{1}{a^{\prime}} \frac{1}{1+q} \mathbf{1}\left\{0 \leq \frac{\ln \left(c / a^{\prime}\right)}{1+q}<(b \wedge \Gamma)\right\}+\frac{\partial m^{*}\left(a^{\prime}, c\right)}{\partial a^{\prime}} \mathbf{1}_{\left\{b \vee 0<m^{*}\left(a^{\prime}, c\right) \leq \Gamma\right\}} \\
& \frac{\partial}{\partial_{-} a^{\prime}} x^{*}\left(a^{\prime}, c, b\right)=-\frac{1}{a^{\prime}} \frac{1}{1+q} \mathbf{1}\left\{0<\frac{\ln \left(c / a^{\prime}\right)}{1+q} \leq(b \wedge \Gamma)\right\}+\frac{\partial m^{*}\left(a^{\prime}, c\right)}{\partial a^{\prime}} \mathbf{1}_{\left\{b \vee 0 \leq m^{*}\left(a^{\prime}, c\right)<\Gamma\right\}}
\end{aligned}
$$


it follows that

$$
\begin{aligned}
\frac{\partial}{\partial_{+/-} a} g\left(a_{T}^{\prime}, c_{T}, b_{T}\right)= & \exp \left(x^{*}\left(a_{T}^{\prime}, c_{T}, b_{T}\right)\right)+a^{\prime} \cdot \exp \left(x^{*}\left(a_{T}^{\prime}, c_{T}, b_{T}\right)\right) \frac{\partial}{\partial_{+/-} a} x^{*}\left(a_{T}^{\prime}, c_{T}, b_{T}\right) \\
= & \exp \left(x^{*}\left(a_{T}^{\prime}, c_{T}, b_{T}\right)\right)-\frac{1}{1+q} \exp \left(x^{*}\left(a_{T}^{\prime}, c_{T}, b_{T}\right)\right) \mathbf{1}_{\{E\}} \\
& +a^{\prime} \cdot \exp \left(x^{*}\left(a_{T}^{\prime}, c_{T}, b_{T}\right)\right) \frac{\partial m^{*}\left(a_{T}^{\prime}, c_{T}\right)}{\partial a^{\prime}} \mathbf{1}_{\{H\}}
\end{aligned}
$$

up to zero sets. Hence, from dominated convergence and (27) we obtain that

$$
\begin{aligned}
\frac{\partial A_{t}^{\prime}}{\partial a_{t}^{\prime}}= & \frac{1}{a_{t}^{\prime}} \mathbb{E}\left[a_{T}^{\prime} \exp \left(x^{*}\left(a_{T}^{\prime}, c_{T}, b_{T}\right)\right)-\frac{1}{1+q} a_{T}^{\prime} \exp \left(x^{*}\left(a_{T}^{\prime}, c_{T}, b_{T}\right)\right) \mathbf{1}_{\{E\}}\right. \\
+ & \left.\left(a_{T}^{\prime}\right)^{2} \cdot \exp \left(x^{*}\left(a_{T}^{\prime}, c_{T}, b_{T}\right)\right) \frac{\partial m^{*}\left(a_{T}^{\prime}, c_{T}\right)}{\partial a^{\prime}} \mathbf{1}_{\{H\}} \mid \mathcal{F}_{t}\right] \\
= & \frac{1}{a_{t}^{\prime}} \mathbb{E}\left[a_{T}^{\prime} \mathbf{1}_{\{D\}} \mid \mathcal{F}_{t}\right]+\frac{q}{1+q} \frac{1}{a_{t}^{\prime}} \mathbb{E}\left[a_{T}^{\prime \frac{q}{1+q}} c_{T}^{\frac{1}{1+q}} \mathbf{1}_{\{E\}} \mid \mathcal{F}_{t}\right] \\
& +\frac{1}{a_{t}^{\prime}} \mathbb{E}\left[a_{T}^{\prime} e^{b_{T}} \mathbf{1}_{\{F\}} \mid \mathcal{F}_{t}\right]+\frac{1}{a_{t}^{\prime}} \mathbb{E}\left[a_{T}^{\prime} e^{\Gamma} \mathbf{1}_{\{G\}} \mid \mathcal{F}_{t}\right]+\frac{1}{a_{t}^{\prime}} \mathbb{E}\left[a_{T}^{\prime} \exp \left(m^{*}\right) \mathbf{1}_{\{H\}} \mid \mathcal{F}_{t}\right] \\
+ & \frac{1}{a_{t}^{\prime}} \mathbb{E}\left[\left(a_{T}^{\prime}\right)^{2} \cdot \exp \left(m^{*}\left(a_{T}^{\prime}, c_{T}\right)\right) \frac{\partial m^{*}\left(a_{T}^{\prime}, c_{T}\right)}{\partial a^{\prime}} \mathbf{1}_{\{H\}} \mid \mathcal{F}_{t}\right]
\end{aligned}
$$

which proves the assertion for the first element in the matrix.

To compute $\partial A_{t}^{\prime} / \partial b_{t}$, notice that $\partial b_{T} / \partial b_{t}=1$ and

$$
\begin{gathered}
\frac{\partial}{\partial_{+} b} x^{*}\left(a^{\prime}, c, b\right)=\mathbf{1}_{\left.\left\{0 \wedge m^{*}\left(a^{\prime}, c\right) \leq b<\Gamma\right)\right\}} \mathbf{1}_{\left\{b<\frac{\ln \left(c / a^{\prime}\right)}{1+q}\right\}} \\
\frac{\partial}{\partial_{-} b} x^{*}\left(a^{\prime}, c, b\right)=\mathbf{1}_{\left.\left\{0 \wedge m^{*}\left(a^{\prime}, c\right)<b \leq \Gamma\right)\right\}} \mathbf{1}_{\left\{b \leq \frac{\ln \left(c / a^{\prime}\right)}{1+q}\right\}} .
\end{gathered}
$$

It follows that the left and right hand derivatives

$$
\begin{aligned}
\frac{\partial}{\partial_{+/-} b} A^{\prime}\left(a_{T}^{\prime}, c_{T}, b_{T}\right) & =a_{T}^{\prime} \cdot \exp \left(x^{*}\left(a_{T}^{\prime}, c_{T}, b_{T}\right)\right) \frac{\partial}{\partial_{+/-} b} x^{*}\left(a_{T}^{\prime}, c_{T}, b_{T}\right) \\
& =a_{T}^{\prime} \exp \left(x^{*}\left(a_{T}^{\prime}, c_{T}, b_{T}\right)\right) \mathbf{1}_{\{F\}}
\end{aligned}
$$

up to zero sets. Hence,

$$
\begin{aligned}
\frac{\partial A_{t}^{\prime}}{\partial b_{t}} & =\mathbb{E}\left[a_{T}^{\prime} \exp \left(x^{*}\left(a_{T}^{\prime}, c_{T}, b_{T}\right)\right) \mathbf{1}_{\{F\}} \mid \mathcal{F}_{t}\right] \\
& =\mathbb{E}\left[a_{T}^{\prime} \exp \left(b_{T}\right) \mathbf{1}_{\{F\}} \mid \mathcal{F}_{t}\right]
\end{aligned}
$$

The other elements are obtained similarly.

The sign of $z_{t}$ is crucial when proving the completeness of the lognormally driven emission market model. The following Lemma implies that $z_{t}$ is negative.

Lemma 3. For fixed $a^{\prime}$ and $c$, the function $b \mapsto A\left(a^{\prime}, c, b\right)$, defined in (13), is monotonically decreasing in $b$ at discontinuities. 
Proof of Lemma 3. The only discontinuities occur at $b=0$ and $b_{T}=\Gamma$. At $b=0$, the discontinuity occurs only on the set $\left\{m^{*}\left(a^{\prime}, c\right) \leq 0\right\}$, then the price jumps from $a^{\prime}+\pi$ to $c$, and because $m^{*}\left(a^{\prime}, c\right) \leq 0$ implies that $a^{\prime}+\pi \geq c$, the jump is decreasing in $b$. If, $m^{*}\left(a^{\prime}, c\right) \leq \Gamma$, the discontinuity at $b=\Gamma$ goes from $a^{\prime} \exp (\Gamma)$ to $c \exp (-q \Gamma)$, only if, $c \exp (-q \Gamma)>a^{\prime} \exp (\Gamma)$. Moreover, if, $\left.m^{*}\left(a^{\prime}, c\right)>\Gamma\right\}$, the price jumps from $a^{\prime} \exp (\Gamma)+\pi$ to $a^{\prime} \exp (\Gamma)$.

The following Proposition states the market completeness, which is a consequence of Lemmas 2 and 3.

Proposition 8. It holds that $\operatorname{det}\left(\Sigma_{t}\right)<0$, almost surely, for all $t \in[0, T)$. Moreover, the lognormally driven emission market model introduced in Definition 3 is complete, if $\operatorname{det}(\Pi) \neq$ 0 .

\section{Proof of Proposition 8.}

The determinant of $\Sigma_{t}$, reads

$$
\begin{array}{r}
\left(-q c_{t} f+z_{t}\right)\left[(a+b+c+d)\left(e+b^{\prime}+f+g\right)-\left(b^{\prime}\right)(b)\right] \\
+q c_{t} f\left[(a+b+c+d)\left(b^{\prime}+f\right)-\left(b^{\prime}\right)(a+b+d)\right] \\
+a_{t}^{\prime} c\left[(b)\left(b^{\prime}+f\right)-\left(e+b^{\prime}+f+g\right)(a+b+d)\right]
\end{array}
$$

and simple algebraic manipulations lead to

$$
\begin{array}{r}
\left(z_{t}\right)\left[(a+c+d)\left(e+b^{\prime}+f+g\right)+(b)(e+f+g)\right] \\
-q c_{t} f\left[(a+b+c+d)(e+g)+\left(b^{\prime}\right)(a+d)\right] \\
-a_{t}^{\prime} c\left[(e+g)(b)+\left(e+b^{\prime}+f+g\right)(a+d)\right] .
\end{array}
$$

It is easy to see that the terms $a, b, b^{\prime}, c, d, e, f$ are non-negative. Due to Lemma 3 , it follows, almost surely, on $[0, T)$ that $z_{t} \leq 0$, and we conclude that the determinant is $\mathbb{Q}$ and almost surely non-positive.

Now let us turn to the proof of market completeness. Due to the non-positivity of the above determinant, we obtain

$$
\begin{aligned}
\mathbb{E}\left[\int_{0}^{T} \mathbf{1}_{\operatorname{det}\left(\Sigma_{t} \Pi\right)=0} d t\right] & =\mathbb{E}\left[\int_{0}^{T} \mathbf{1}_{\operatorname{det}\left(\Sigma_{t}\right)=0} d t\right]=\mathbb{E}\left[\lim _{n \rightarrow \infty} \int_{0}^{T-1 / n} \mathbf{1}_{\operatorname{det}\left(\Sigma_{t}\right)=0} d t\right] \\
& =\lim _{n \rightarrow \infty} \mathbb{E}\left[\int_{0}^{T-1 / n} \mathbf{1}_{\operatorname{det}\left(\Sigma_{t}\right)=0} d t\right]=\lim _{n \rightarrow \infty} \int_{0}^{T-1 / n} \mathbb{P}\left[\operatorname{det}\left(\Sigma_{t}\right)=0\right] d t=0
\end{aligned}
$$

where the first equality holds, because $\mathbf{1}_{\operatorname{det}\left(\Sigma_{T}\right)=0} \leq 1$. Moreover, the second equality holds due to dominated convergence, since $\int_{0}^{T-1 / n} \mathbf{1}_{\operatorname{det}\left(\Sigma_{t}\right)=0} d t<T$, and the third equality holds due to the Fubinis Theorem, because $\Sigma_{t}$ is adapted, and continuous on $[0, T)$, and, hence, $\left(\operatorname{det}\left(\Sigma_{t}\right)\right)_{t \in[0, T)}$ product-measurable on $\Omega \times[0, T)$. The inclusion follows directly from (33).

Therefore, applying the standard results of mathematical finance, the price at any time $0 \leq t \leq$ $T$, of a spread option with payoff $\left(A_{T}-C_{T}\right)^{+}$, is uniquely determined as: Proof of Proposition 
7. For an arbitrage free emission market, in the sense of Definition 2, equation (16) implies that $\left\{A_{T}>C_{T}\right\}=\left\{A_{T}^{\prime}>C_{T}\right\}$ holds on $N^{c}$. Hence, if the probability for noncompliance is small, as it is for the EU ETS, we can approximate the spread option price between Phase II EUA and CER by the spread option price of Phase III EUA and CER:

$$
B_{t} \mathbb{E}\left[\frac{\left(A_{T}-C_{T}\right)^{+}}{B_{T}} \mid \mathcal{F}_{t}\right] \approx B_{t} \mathbb{E}\left[\frac{\left(A_{T}^{\prime}-C_{T}\right)^{+}}{B_{T}} \mid \mathcal{F}_{t}\right] .
$$

For any emission market set up as in Proposition 4, it holds that $\left\{A_{T}^{\prime}>C_{T}\right\} \subseteq\left\{A_{T}^{\prime}=\right.$ $\left.a_{T}^{\prime}\right\} \cap\left\{C_{T}=c_{T}\right\}$ and $\left\{A_{T}^{\prime} \leq C_{T}\right\} \subseteq\left\{a_{T}^{\prime} \leq c_{T}\right\}$, which is due to the parameterization introduced in Section 3.3. Using this we obtain

$$
B_{t} \mathbb{E}\left[\frac{\left(A_{T}^{\prime}-C_{T}\right)^{+}}{B_{T}} \mid \mathcal{F}_{t}\right]=B_{t} \mathbb{E}\left[\frac{\left(a_{T}^{\prime}-c_{T}\right)^{+}}{B_{T}} \mid \mathcal{F}_{t}\right]
$$

Moreover, assuming a deterministic short rate $r$ in connection with the lognormal emission market, the spread option price reduces to margrabes formula, expressed in terms of $a_{t}^{\prime}$ and $c_{t}$, instead of $A_{t}^{\prime}$ and $C_{t}$. Notice that for the last equation of Proposition 7, we used that the correlation between $W^{a}$ and $W^{c}$, which is assumed to be 0 .

\section{Proof of Model Tractability}

Proof of Proposition 5. For notational simplicity, we restrict ourselves to the time point $t=0$, where $a_{0}^{\prime}, b_{0}, c_{0} \in \mathbb{R}$ is almost certain. Since $\left(a_{t}^{\prime}\right)_{t \in[0, T]},\left(b_{t}\right)_{t \in[0, T]}$ and $\left(c_{t}\right)_{t \in[0, T]}$ are Markov processes under $\mathbb{F}$, measurable functions $\mathrm{A}, \mathrm{A}^{\prime}, \mathrm{C}: \mathbb{R}^{3} \rightarrow \mathbb{R}$ exist with $\mathrm{A}\left(a_{0}^{\prime}, b_{0}, c_{0}\right)=A_{0}=$ $\mathbb{E}\left[A_{T} \mid \mathcal{F}_{0}\right], \mathrm{A}^{\prime}\left(a_{0}^{\prime}, b_{0}, c_{0}\right)=A_{0}^{\prime}$ and $\mathrm{C}\left(a_{0}^{\prime}, b_{0}, c_{0}\right)=C_{0}$. The precise form, of which, will be derived in the Appendix.

Since $\left(C_{t}\right)_{t \in[0, T]}$ and $\left(c_{t}\right)_{t \in[0, T]}$ are martingales, it follows from $c_{T} \exp (-q \Gamma) \leq C_{T} \leq c_{T}$ that $c_{0} \exp (-q \Gamma) \leq C_{0} \leq c_{0}$, and equivalently $\mathrm{C}\left(a_{0}^{\prime}, b_{0}, c_{0}\right) \leq c_{0} \leq \mathrm{C}\left(a_{0}^{\prime}, b_{0}, c_{0} \exp (q \Gamma)\right)$, are almost certain for all $a_{0}^{\prime}, b_{0} \in \mathbb{R}$. Since for fixed $a_{0}^{\prime}$ and $b_{0}$, the function $c_{0} \mapsto C_{0}\left(a_{0}^{\prime}, b_{0}, c_{0}\right)$ is continuous and monotone increasing, it follows from the intermediate value theorem, that a unique $c_{0}^{*} \in\left[C_{0}^{*}, C_{0}^{*} \exp (q \Gamma)\right]$ with $\mathrm{C}\left(a_{0}^{\prime}, c_{0}^{*}, b_{0}\right)=C_{0}^{*}$ exists. Therefore, let us denote this unique solution by $c^{*}\left(a_{0}^{\prime}, b_{0}\right)$.

The same argument holds for $a_{0}^{\prime}$, and for all $b_{0}, a_{0}^{\prime}$ we have $a_{0}^{\prime} \leq \mathrm{A}^{\prime}\left(a_{0}^{\prime}, c^{*}\left(a_{0}^{\prime}, b_{0}\right), b_{0}\right) \leq$ $a_{0}^{\prime} \exp (p \Gamma)$ or equivalently $\mathrm{A}^{\prime}\left(a_{0}^{\prime}, c^{*}\left(a_{0}^{\prime}, b_{0}\right), b_{0}\right) \leq a_{0}^{\prime} \leq \mathrm{A}^{\prime}\left(a_{0} \exp (-p \Gamma), c^{*}\left(a_{0}^{\prime} \exp (-p \Gamma), b_{0}\right), b_{0}\right)$. Since $a_{0}^{\prime} \mapsto \mathrm{A}\left(a_{0}^{\prime}, c^{*}\left(a_{0}^{\prime}, b_{0}\right), b_{0}\right)$ is continuous, it follows from the intermediate value theorem that there is an $a_{0}^{\prime *} \in\left[A_{0}^{\prime *} \exp (-p \Gamma), A_{0}^{\prime *}\right]$, such that $\mathrm{A}^{\prime}\left(a_{0}^{\prime *}, c^{*}\left(a_{0}^{\prime *}, b_{0}\right), b_{0}\right)=A_{0}^{\prime *}$; this value is unique if the function $a_{0}^{\prime} \mapsto \mathrm{A}^{\prime}\left(a_{0}^{\prime}, c^{*}\left(a_{0}^{\prime}, b_{0}\right), b_{0}\right)$ is monotone increasing.

To prove this assumption, that, on the contrary, for fixed $b_{0}$ there exists a maximum of $a_{0}^{\prime} \mapsto \mathrm{A}^{\prime}\left(a_{0}^{\prime}, c\left(a_{0}^{\prime}, b_{0}\right), b_{0}\right)$, then there is also a maximum of $\left(a_{0}^{\prime}, c_{0}\right) \rightarrow \mathrm{A}^{\prime}\left(a_{0}^{\prime}, c_{0}, b_{0}\right)$ under the constraint $\mathrm{C}\left(a_{0}^{\prime}, c_{0}, b_{0}\right)=C_{0}^{*}$. However, since

$$
\operatorname{det}\left(\begin{array}{ll}
\frac{\partial \mathrm{A}^{\prime}}{\partial a_{0}^{\prime}} & \frac{\partial \mathrm{A}^{\prime}}{\partial c_{0}} \\
\frac{\partial \mathcal{C}}{\partial a_{0}^{\prime}} & \frac{\partial \mathrm{C}}{\partial c_{0}}
\end{array}\right)>0
$$


and the derivatives in the matrix are continuously differentiable on an open subset of $\mathbb{R}^{3}$, it follows from Lagrange's Theorem that there is no point at which the optimum is attained, proving the monotonicity, and, hence, the uniqueness of $a_{0}^{\prime}$. Thus, for a given $b_{0}$, there is a unique pair $\left(a^{*}\left(b_{0}\right), c^{*}\left(b_{0}\right)\right)$, such that $\mathrm{A}^{\prime}\left(a^{*}\left(b_{0}\right), c^{*}\left(b_{0}\right), b_{0}\right)=A^{* *}$ and $\mathrm{C}\left(a^{*}\left(b_{0}\right), c^{*}\left(b_{0}\right), b_{0}\right)=$ $C_{0}^{*}$.

Moreover, it is clear that $\lim _{b_{0} \rightarrow \infty} \mathrm{A}\left(a^{*}\left(b_{0}\right), c^{*}\left(b_{0}\right), b_{0}\right)=A^{* *}$ and $\lim _{b_{0} \rightarrow-\infty} \mathrm{A}\left(a^{*}\left(b_{0}\right), c^{*}\left(b_{0}\right), b_{0}\right)$ $=A^{\prime *}+\pi$. Due to Proposition 8, it holds that $\Sigma_{t}$, defined in (30), is almost surely non-positive. Hence, due to the Lagrange Theorem, we, again, conclude that $b_{0} \mapsto \mathrm{A}\left(a^{*}\left(b_{0}\right), c^{*}\left(b_{0}\right), b_{0}\right)$ is monotone decreasing, and we know that there is a unique triple $\left(a_{0}^{\prime *}, c_{0}^{*}, b_{0}^{*}\right)$, such that the model is calibrated.

\section{Calibration of Model Parameters to Phase II of EU ETS}

In this Section, we discuss the Calibration of the model with the simpler correlation structure, as discussed in subsection 4.2 for Phase II of the EU ETS. In subsection D.1, we use historical emission data to calibrate $b_{0}$ and $\sigma_{b}$. In subsection D.2, we use historical price data to calibrate $\sigma_{a}, \sigma_{c}$ and $p / q$; whilst in subsection D.3 we show that under some assumptions, the parameter $\rho_{a, b}$ is not a free model parameter, but is given by $\sigma_{a}, \sigma_{b}$ and $p$. In subsection D.4, we show how the different estimates are used to calibrate the model when pricing the spread option for different $p$. Moreover, subsection D.5, provides some interesting insights into the correlation structure of the model and its dependence on $p$ and $q$.

\section{D.1 Calibration of $\sigma_{b}$ and $b_{0}$}

The projected emission under $\mathbb{Q}$ is given by $\mathbb{E}_{\mathbb{Q}}\left[\mathcal{E}_{T} \mid \mathcal{F}_{0}\right]=b_{0}+\Lambda$. Hence, for our model framework, $b_{0}$ should be calibrated implicitly to price data. However, if $A_{0} \approx A_{0}^{\prime}$, as in Phase II of the EU ETS, this estimation is very unstable.

Hence, a crude approximation is made, and we assume that under $\mathbb{Q}$, the expected emission $\mathbb{E}_{\mathbb{Q}}\left[\mathcal{E}_{T} \mid \mathcal{F}_{0}\right]$, up to the end of the compliance period, is given, approximately, by projecting the average emission from 2008 and 2009 for the rest of the period. An estimate for this $b_{0}$ is easy to find, since the import limit $\Gamma$ and the initial allocation $\Lambda$ (up to a small allocation reserve) are specified in the national allocation plans. Moreover, we estimate $\sigma_{b}$, by computing the standard deviation of the yearly emission data from 2005 to 2009. The corresponding emission and allocation data is displayed in Table 35, while the import limit is given by $\Gamma=1.4 G t$. Putting these together, yields: $\sigma_{b}=0.09 G t / a$ and $b_{0}=1.1 G t$.

\begin{tabular}{|c|c|c|}
\hline \multicolumn{3}{|c|}{ EU ETS verified emission and allocation. } \\
\hline Year & Verified Emission & Allocation \\
\hline 2005 & $2012 \mathrm{Mt}$ & $2151 \mathrm{Mt}$ \\
\hline 2006 & $2033 \mathrm{Mt}$ & $2151 \mathrm{Mt}$ \\
\hline 2007 & $2049 \mathrm{Mt}$ & $2151 \mathrm{Mt}$ \\
\hline 2008 & $2119 \mathrm{Mt}$ & $1957 \mathrm{Mt}$ \\
\hline 2009 & $1873 \mathrm{Mt}$ & $1957 \mathrm{Mt}$ \\
\hline
\end{tabular}




\section{D.2 Calibration of $\sigma_{a}, \sigma_{c}$ and $p / q$}

In this section we calibrate $\sigma_{a}, \sigma_{c}$, and the ratio of $p$ and $q$, to historical data for EU ETS. As has been discussed, the probability for the events $F, G, H$ and $N$ vanish in the EU ETS, and it makes perfect sense to use the reduced model from Section 4.4. In this setting, it holds that $a^{\prime \frac{q}{p+q}} c^{\frac{p}{p+q}}=a^{\prime \mu} c^{1-\mu}$ with $p / q=\mu /(1-\mu)$, and the model is invariant for a constant price response ratio, $p / q$, between CERs and successive period's EUAs. Hence, we can only estimate this ratio $p / q$, instead of $p$ and $q$. Calibrating these parameters to EUA and CER futures price processes with a maturity December 2012 gives the following maximum likelihood estimates for the time period $14 / 3 / 2008$ to $24 / 8 / 2010: \sigma_{a}=0.34, \sigma_{c}=0.32$ and $p / q=0.66$.

\section{D.3 Calibration of $\rho_{a, b}$}

Until now, all parameters have been calibrated with the exception of $p$ and $\rho_{a, b}$. In Subsection 5.3, $p$ is used as a free parameter in order to investigate its impact upon spread option prices. Hence, we do not need to calibrate it, and, therefore, the only remaining parameter to be calibrated is $\rho_{a, b}$. To this end, we make the crude assumption that in the compliance situation $F$, where no EUAs are banked to the next period, the Phase III EUA price should be uncorrelated with emissions in Phase II. In particular, this assumption makes sense if there is no feedback of CER prices for next period's EUA prices. Under this assumption, $\rho_{a, b}$ is not a free model parameter, but, in fact, is dependent on $\sigma_{a}, \sigma_{b}$ and $p$. This can be demonstrated as follows: On $F$, it holds that $A_{T}^{\prime}=a_{T}^{\prime} \exp \left(p b_{T}\right)$, which reduces to $a_{T}^{\prime} \exp \left(p b_{T}\right)=a_{0} \exp \left(\sigma_{a} W_{T}^{a}-\frac{1}{2} \sigma_{a}^{2} T\right) \exp \left(p\left(b_{0}+\sigma_{b} W_{T}^{b}\right)\right)$, while $W^{a}$ and $W^{b}$ are correlated by $\rho_{a, b}$. Hence, for $A_{T}^{\prime}$ and $b_{T}$ to be uncorrelated, it is required that $\rho_{a, b}=-p \sigma_{b} / \sigma_{a}$, which fixes $\rho_{a, b}$ for given $\sigma_{a}, \sigma_{b}$ and $p$.

\section{D.4 Calibration of the Spread Option}

The initial futures curve is calibrated to the EU ETS prices observed on 24/8/2010, i.e. $A_{0}^{\prime}=16$ and $C_{0}=13$. The spread option we consider has a maturity of $31 / 12 / 2012$.

Note that the model is no longer invariant with respect to the constant $\mu=p /(p+q)$, and, therefore, it is important to look at the impact of adjusting the price response parameters $p, q$, in such a way that the ratio $\mu=p /(p+q)$ of the CER and EUA price response is constant. For every value of the parameter $p$, we have to recalibrate $a_{0}^{\prime}$ and $c_{0}$ to match the initial futures prices $A_{0}^{\prime *}=16$ and $C_{0}^{*}=13$, but we also have to adjust $\rho_{a, b}$, such that $\rho_{a, b}=-\sigma_{b} /\left(p \sigma_{a}\right)$. Since $\rho_{a, b}$ is constrained by $\rho_{a, b} \in(-1,1)$, the price response parameter must fulfill the condition $p>\sigma_{b} / \sigma_{a}=0.42$.

\section{D.5 Interpretation of Correlation Structure}

The correlation matrix $\Pi$ and the parameters $p, q$ fix the correlation structure of phase III EUA prices, CER prices and emissions. In particular, at the end of the compliance period there are two situations that characterize these parameters: In the first case, all CERs are used for compliance, and $A_{T}^{\prime}=a_{T}^{\prime}$, as well as $C_{T}=c_{T}$. Here, the correlation between the log 
prices and emissions are given directly by the correlation matrix.

In the second case, where only the compliance gap $\mathcal{E}_{T}-\Lambda$ is covered by CERs, the prices read $A_{T}^{\prime}=a_{T}^{\prime} \exp \left(p b_{T}\right)$ and $C_{T}=c_{T} \exp \left(-q b_{T}\right)$. Here, the correlation structure is totally different, and the change in correlation is governed by $p$ and $q$.

The economic interpretation is: In the first case, emissions drive the amount of Phase III EUAs banked to the next period; in the second case, emissions drive the amount of CERs carried over. Hence, in one case emissions drive the Phase III EUA price, and in the other, the CER price.

\section{E Closed Form Formulae for the Prices}

In this subsection, we focus on deriving some closed form formulae for the prices i.e. we evaluate the conditional expectations for the EUA and CER prices up to one dimensional integral.

For a real market, it seems very unlikely that $C_{T} \geq A_{T}^{\prime}+\pi$, thus, we evaluate the conditional expectations under the assumption that $m^{*} \geq \Gamma$. Since these formulas were used to generate the price scenarios in the preceding section, we restrict ourselves to the simple correlation structure, where only $W^{a}$ and $W^{b}$ are correlated by a constant $\rho_{a, b}$. Equations (27) -(29) state that our price processes are sums of products of GBM (with a potential drift), and the indicator function for one of our five market situations. Hence, it is clear that by applying Girsanov's Theorem, we can express the conditional expectation of each summand in closed form up to the function

$$
\varphi(e, f, g, h)=\frac{1}{\sqrt{2 \pi}} \int_{e}^{f} \exp \left(-x^{2} / 2\right) Q(g+h x) d x=\frac{1}{2 \pi} \int_{e}^{f} \exp \left(-x^{2} / 2\right) \int_{g+h x}^{\infty} \exp \left(-y^{2} / 2\right) d y d x .
$$

Since this involves only a one dimensional integral, computations are fast enough for practical applications; the precise result is:

Proposition 9. $s_{t}=\left(a_{t}^{\prime}, c_{t}, b_{t}, t\right)$ denotes the state of the market at time $t \in[0, T]$, and $g_{B}(t)$, $g_{C}(t), g_{C}^{\prime}(t), \varphi_{B}\left(s_{t}\right), \varphi_{C}\left(s_{t}\right), \varphi_{C}^{\prime}\left(s_{t}\right), \varphi_{D}\left(s_{t}\right)$, and $\varphi_{D}\left(s_{t}\right)$ denotes the functions defined in the following Lemmas 4 - 8. If $\left(a^{\prime}\right)_{t \in[0, T]},(c)_{t \in[0, T]}$, and $(b)_{t \in[0, T]}$ are given by $(21)$, then the next period's allowance and CER price processes are, almost surely,

$$
\begin{aligned}
& A_{t}^{\prime}=a_{t}^{\prime}\left(1-\varphi_{D}\left(0, s_{t}\right)\right)+\left(c_{t}\right)^{\frac{p}{p+q}}\left(a_{t}^{\prime}\right)^{\frac{q}{p+q}} g_{B}(t) \varphi_{B}\left(s_{t}\right)+a_{t}^{\prime} e^{p b_{t}} g_{C}(t) \varphi_{C}\left(s_{t}\right)+a_{t}^{\prime} e^{p \Gamma} \varphi_{D}\left(\kappa, s_{t}\right) \\
& C_{t}=c_{t}\left(1-\varphi_{D}\left(0, s_{t}\right)\right)+\left(c_{t}\right)^{\frac{p}{p+q}}\left(a_{t}^{\prime}\right)^{\frac{q}{p+q}} g_{B}(t) \varphi_{B}\left(s_{t}\right)+c_{t} e^{-q b_{t}} g_{C}^{\prime}(t) \varphi_{C}^{\prime}\left(s_{t}\right)+c_{t} e^{-q \Gamma} \varphi_{D}^{\prime}\left(\kappa, s_{t}\right) .
\end{aligned}
$$

Moreover, the ongoing periods allowance price process is given by, almost surely,

$$
A_{t}=A_{t}^{\prime}+\phi\left(\frac{-b_{t}}{\sigma_{b} \sqrt{T-t}}\right)+c_{t} e^{-q b_{t}} g_{C}^{\prime}(t) \varphi_{C}^{\prime}\left(s_{t}\right)-a_{t}^{\prime} e^{p b_{t}} g_{C}(t) \varphi_{C}\left(s_{t}\right)
$$

The proof of this Proposition requires Lemmas $4-8$, which are established after the proof. Since their proofs are straight forward and involve only simple measure changes, relying on Girsanov's Theorem, we do not provide the particulars. 
Proof of Proposition 9. the next periods allowance price processes are a direct consequence of Lemma $4-8$ and the identity

$$
\mathbb{E}\left[a_{T}^{\prime} \mathbf{1}_{\{A\}} \mid \mathcal{F}_{t}\right]=\mathbb{E}\left[a_{T}^{\prime}-a_{T}^{\prime} \mathbf{1}_{\left\{b_{T} \geq 0\right\}} \mathbf{1}_{\left\{\ln \left(c_{T} / a_{T}^{\prime}\right) \geq 0\right\}} \mid \mathcal{F}_{t}\right]=a_{t}^{\prime}-a_{t}^{\prime} \varphi_{D}\left(0, s_{t}\right) .
$$

The ongoing period's allowance price follows from Lemmas 6,5 and

$$
\begin{aligned}
A_{t}= & A_{t}^{\prime}+\pi \mathbb{E}_{\mathbb{Q}}\left[\mathbf{1}_{\left\{b_{T}<0\right\}} \mid \mathcal{F}_{t}\right]+\mathbb{E}_{Q}\left[\left(c_{T}\right) \exp \left(-q b_{T}\right) \mathbf{1}_{\left\{0 \leq b_{T} \leq \Gamma\right\}} \mathbf{1}_{\left\{\ln \left(c_{T} / a_{T}^{\prime}\right) \geq b_{T}(p+q)\right\}} \mid \mathcal{F}_{t}\right] \\
& -\mathbb{E}_{Q}\left[\left(a_{T}^{\prime}\right) \exp \left(p b_{T}\right) \mathbf{1}_{\left\{0 \leq b_{T} \leq \Gamma\right\}} \mathbf{1}_{\left\{\ln \left(c_{T} / a_{T}^{\prime}\right) \geq b_{T}(p+q)\right\}} \mid \mathcal{F}_{t}\right] \\
= & A_{t}^{\prime}+\pi \phi\left(\frac{-b_{t}}{\sigma_{b} \sqrt{T}}\right)+c_{t} e^{-q b_{t}} g_{C}^{\prime}(t) \varphi_{C}^{\prime}\left(s_{t}\right)-a_{t}^{\prime} e^{p b_{t}} g_{C}(t) \varphi_{C}\left(s_{t}\right)
\end{aligned}
$$

almost surely.

Lemma 4. If, $a^{\prime}, c$ and $b$ are given by (21), it then holds that

$$
\mathbb{E}_{\mathbb{Q}}\left[\left(c_{T}\right)^{\frac{p}{p+q}}\left(a_{T}^{\prime}\right)^{\frac{q}{p+q}} \mathbf{1}_{\{B\}} \mid \mathcal{F}_{t}\right]=\left(c_{t}\right)^{\frac{p}{p+q}}\left(a_{t}^{\prime}\right)^{\frac{q}{p+q}} g_{B}(t) \varphi_{B}\left(a_{t}^{\prime}, c_{t}, b_{t}, t\right)
$$

almost surely, with

$$
\begin{aligned}
g_{B}(t) & =\exp \left(\frac{1}{2}\left(\left(\frac{p}{p+q}\right)^{2}-\frac{p}{p+q}\right) \sigma_{c}^{2}(T-t)+\frac{1}{2}\left(\left(\frac{q}{p+q}\right)^{2}-\frac{q}{p+q}\right) \sigma_{a}^{2}(T-t)\right) \\
\varphi_{B}\left(a_{t}^{\prime}, c_{t}, b_{t}, t\right) & =\varphi\left(e\left(a_{t}^{\prime}, c_{t}, t\right), f(t), g\left(a_{t}^{\prime}, c_{t}, b_{t}, t\right), h\right)
\end{aligned}
$$

and

$$
\begin{aligned}
e\left(a_{t}^{\prime}, c_{t}, t\right) & =\frac{-\ln \left(\frac{c_{t}}{a_{t}^{\prime}}\right)-\frac{1}{2} \frac{p-q}{p+q}\left(\sigma_{a}^{2}(T-t)+\sigma_{c}^{2}(T-t)\right)}{\sqrt{\sigma_{c}^{2}(T-t)+\sigma_{a}^{2}(T-t)}} \\
g\left(a_{T}^{\prime}, c_{t}, b_{t}, t\right) & =\frac{\ln \left(\frac{c_{t}}{a_{t}^{\prime}}\right)+\frac{1}{2} \frac{p-q}{p+q}\left(\sigma_{a}^{2}(T-t)+\sigma_{c}^{2}(T-t)\right)-b_{t}(p+q)-q \sigma_{a} \sigma_{b} \rho(T-t)}{\sqrt{\left(1-\frac{\sigma_{a}^{2} \rho^{2}}{\sigma_{c}^{2}+\sigma_{a}^{2}}\right)} \sigma_{b}(p+q) \sqrt{T-t}} \\
h & =\frac{\sigma_{c}^{2}+\sigma_{a}^{2}+\sigma_{b} \sigma_{a} \rho(p+q)}{\sqrt{\sigma_{c}^{2}+\sigma_{a}^{2}\left(1-\rho^{2}\right)} \sigma_{b}(p+q)} \quad f(t)=e+\frac{\Gamma(p+q)}{\sqrt{\sigma_{c}^{2}(T-t)+\sigma_{a}^{2}(T-t)}} .
\end{aligned}
$$

Lemma 5. If $a^{\prime}, c$ and $b$ are given by (21), it then holds, that

$$
\mathbb{E}_{\mathbb{Q}}\left[\left(a_{T}^{\prime}\right) \exp \left(p b_{T}\right) \mathbf{1}_{\left\{0 \leq b_{T} \leq \Gamma\right\}} \mathbf{1}_{\left\{\ln \left(c_{T} / a_{T}^{\prime}\right) \geq b_{T}(p+q)\right\}} \mid \mathcal{F}_{t}\right]=\left(a_{t}^{\prime}\right) \exp \left(p b_{t}\right) g_{C}(t) \varphi_{C}\left(a_{t}^{\prime}, c_{t}, b_{t}, t\right)
$$

almost surely, with

$$
g_{C}(t)=\exp \left(\frac{1}{2} p^{2} \sigma_{b}^{2}(T-t)+p \rho \sigma_{a} \sigma_{b}(T-t)\right) \quad \varphi_{C}\left(a_{t}^{\prime}, c_{t}, b_{t}, t\right)=\varphi\left(e\left(b_{t}, t\right), f(t), g\left(a_{t}^{\prime}, c_{t}, t\right), h\right)
$$

and

$$
\begin{aligned}
e\left(b_{t}, t\right) & =\frac{-b_{t}-p \sigma_{b}^{2}(T-t)-\sigma_{a} \sigma_{b} \rho(T-t)}{\sigma_{b} \sqrt{T-t}} \quad f(t)=e+\frac{\Gamma}{\sigma_{b} \sqrt{T-t}} \quad h=\frac{\left((p+q) \sigma_{b}+\sigma_{a} \rho\right)}{\sqrt{\sigma_{c}^{2}+\sigma_{a}^{2}\left(1-\rho^{2}\right)}} \\
g\left(a_{t}^{\prime}, c_{t}, t\right) & =\frac{b_{t}(p+q)+p(p+q) \sigma_{b}^{2}(T-t)-\ln \left(\frac{c_{t}}{a_{t}^{\prime}}\right)+\frac{1}{2}\left(\sigma_{a}^{2}(T-t)+\sigma_{c}^{2}(T-t)\right)+(2 p+q) \sigma_{a} \rho \sigma_{b} T}{\sqrt{\sigma_{c}^{2}(T-t)+\sigma_{a}^{2}\left(1-\rho^{2}\right)(T-t)}} .
\end{aligned}
$$


The following Lemma is a direct consequence of Lemma 5:

Lemma 6. If, $a^{\prime}, c$ and $b$ are given by (21), it then holds, that

$$
\mathbb{E}_{\mathbb{Q}}\left[\left(c_{T}\right) \exp \left(-q b_{T}\right) \mathbf{1}_{\left\{0 \leq b_{T} \leq \Gamma\right\}} \mathbf{1}_{\left\{\ln \left(c_{T} / a_{T}^{\prime}\right) \geq b_{T}(p+q)\right\}} \mid \mathcal{F}_{t}\right]=\left(c_{t}\right) \exp \left(-q b_{t}\right) g_{C}^{\prime}(t) \varphi_{C}^{\prime}\left(a_{t}^{\prime}, c_{t}, b_{t}, t\right)
$$

almost surely, with

$$
g_{C}^{\prime}(t)=\exp \left(\frac{1}{2} q^{2} \sigma_{b}^{2}(T-t)\right) \quad \varphi_{C}^{\prime}\left(a_{t}^{\prime}, c_{t}, b_{t}, t\right)=\varphi\left(e\left(b_{t}, t\right), f(t), g\left(a_{t}^{\prime}, c_{t}, t\right), h\right)
$$

and

$$
\begin{aligned}
e\left(b_{t}, t\right) & =\frac{-b_{t}+q \sigma_{b}^{2}(T-t)}{\sigma_{b} \sqrt{T-t}} \quad f(t)=e+\frac{\Gamma}{\sigma_{b} \sqrt{T-t}} \quad h=\frac{\left((p+q) \sigma_{b}+\sigma_{a} \rho\right)}{\sqrt{\sigma_{c}^{2}+\sigma_{a}^{2}\left(1-\rho^{2}\right)}} \\
g\left(a_{t}^{\prime}, c_{t}, t\right) & =\frac{(p+q) b_{t}-(p+q) q \sigma_{b}^{2}(T-t)-\ln \left(\frac{c_{t}}{a_{t}^{\prime}}\right)-\frac{1}{2}\left(\sigma_{a}^{2}(T-t)+\sigma_{c}^{2}(T-t)\right)-q \sigma_{a} \rho \sigma_{b}(T-t)}{\sqrt{\sigma_{c}^{2}(T-t)+\sigma_{a}^{2}\left(1-\rho^{2}\right)(T-t)}} .
\end{aligned}
$$

Lemma 7. Let $z \in \mathbb{R}$. If $a^{\prime}, c$ and $b$ are given by (21) it then holds, almost surely, that

$$
\mathbb{E}\left[a_{T}^{\prime} \mathbf{1}_{\left\{b_{T} \geq z\right\}} \mathbf{1}_{\left\{\ln \left(c_{T} / a_{T}^{\prime}\right) \geq z(p+q)\right\}} \mid \mathcal{F}_{t}\right]=a_{t}^{\prime} \varphi_{D}\left(z, a_{t}^{\prime}, c_{t}, b_{t}, t\right)
$$

with

$$
\begin{aligned}
& \varphi_{D}\left(z, a_{t}^{\prime}, c_{t}, b_{t}, t\right)=\varphi\left(e\left(z, b_{t}, t\right), \infty, g\left(z, a_{t}^{\prime}, c_{t}, t\right), h\right) \quad e\left(z, b_{t}, t\right)=\frac{z-b_{t}-\rho \sigma_{a} \sigma_{b}(T-t)}{\sigma_{b} \sqrt{(T-t)}} \\
& g\left(z, a_{t}^{\prime}, c_{t}, t\right)=\frac{z(p+q)-\ln \left(\frac{c_{t}}{a_{t}^{\prime}}\right)+\frac{1}{2}\left(\sigma_{a}^{2}(T-t)+\sigma_{c}^{2}(T-t)\right)}{\sqrt{\sigma_{c}^{2}(T-t)+\sigma_{a}^{2}\left(1-\rho^{2}\right)(T-t)}} \quad h=\frac{\sigma_{a} \rho}{\sqrt{\sigma_{c}^{2}+\sigma_{a}^{2}\left(1-\rho^{2}\right)}}
\end{aligned}
$$

The following Lemma is a direct consequence of Lemma 7:

Lemma 8. Let $z \in \mathbb{R}$. If, $a^{\prime}, c$ and $b$ are given by (21), it then holds, that

$$
\mathbb{E}_{\mathbb{Q}}\left[c_{T} \mathbf{1}_{\left\{b_{T} \geq z\right\}} \mathbf{1}_{\left\{\ln \left(c_{T} / a_{T}^{\prime}\right) \geq z(p+q)\right\}} \mid \mathcal{F}_{t}\right]=c_{t} \varphi_{D}^{\prime}\left(z, a_{t}^{\prime}, c_{t}, b_{t}, t\right)
$$

with

$$
\begin{aligned}
\varphi_{D}^{\prime}\left(z, a_{t}^{\prime}, c_{t}, b_{t}, t\right) & =\varphi\left(e\left(z, b_{t}, t\right), \infty, g\left(z, a_{t}^{\prime}, c_{t}, t\right), h\right) \quad h=\frac{\sigma_{a} \rho \sqrt{(T-t)}}{\sqrt{\sigma_{c}^{2}(T-t)+\sigma_{a}^{2}\left(1-\rho^{2}\right)(T-t)}} \\
g\left(z, a_{t}^{\prime}, c_{t}, t\right) & =\frac{z(p+q)-\ln \left(\frac{c_{t}}{a_{t}^{\prime}}\right)-\frac{1}{2}\left(\sigma_{a}^{2}(T-t)+\sigma_{c}^{2}(T-t)\right)}{\sqrt{\sigma_{c}^{2}(T-t)+\sigma_{a}^{2}\left(1-\rho^{2}\right)(T-t)}} \quad e\left(z, b_{t}, t\right)=\frac{z-b_{t}}{\sigma_{b} \sqrt{(T-t)}}
\end{aligned}
$$

\section{References}

[Bloch(2010)] Bloch, D. A. 2010. A Note On Emissions Trading: The Pricing Of Carbon Derivatives. Working Paper, Universit Paris VI Pierre et Marie Curie, Paris.

[Carmona et al.(2010)] Carmona, R., Fehr, M., Hinz, J., Porchet, A. 2010. Market Designs for Emissions Trading Schemes. SIAM Review. 52(3) 403-452. 
[Carmona et al.(2009)] Carmona, R., Fehr, M., Hinz, J. 2009. Optimal stochastic control and carbon price formation. SIAM Journal on Control and Optimization. 48(12) 2168-2190.

[Carmona et al.(2008)] Carmona, R., Fehr, M., Hinz, J. 2008. Properly Designed Emission Trading Schemes Do Work. Working Paper. Princeton University, Princeton.

[Carmona and Fehr(2010)] Carmona, R., Fehr, M. 2010. The Clean Development Mechanism and Price Formation for Allowances and CERs. Progress in Probability. 63 341-383.

[Carmona and Hinz(2009)] Carmona, R., Hinz, J. 2009. Risk Neutral Modeling of Emission Allowance Prices and Option Valuation. Working paper, Princeton University, Princeton.

[Cetin and Verschuere(2010)] Cetin, U., Verschuere, M. 2010. Pricing and hedging in carbon emissions markets. International Journal of Theoretical and Applied Finance 12(7) 949-967.

[Chesney and Taschini(2008)] Chesney, M., Taschini, L. 2008. The endogenous price dynamics of the emission allowances: An application to co2 option pricing. Working Paper, University of Zurich, Zurich.

[Cronshaw and Kruse(1996)] Cronshaw, M., Kruse, J. B. 1996. Regulated Firms in Pollution Permit Markets with Banking Journal of Regulatory Economics. 9(2) 179-189.

[Dales(1968)] Dales, J. H. 1968. Pollution, Property and Prices. University of Toronto Press, Toronto.

[Gruell and Taschini (2010)] Gruell, G., Taschini, L. 2010. A Comparison of Reduced-Form Permit Price Models and their Empirical Performances. Working Paper, London School of Economics, London.

[Hinz and Fehr(2010)] Hinz, J., Fehr, M. 2010. Storage costs in commodity option pricing. Siam Journal on Financial Mathematics. 1 729-751.

[Lewis and Curien(2009)] Lewis, M.C., Curien, I. 2009. Chapter \& Verse: EU-ETS Rules for CER/ERU Use Beyond Copenhagen. Global Markets Research, Deutsche Bank.

[Montgomery(1972)] Montgomery, W. D. 1972. Markets in Licenses and Efficient Pollution Control Programs Journal of Economic Theory. 5(3) 395-418.

[Rubin(1996)] Rubin, J. 1996. A Model of Intertemporal Emission Trading, Banking and Borrowing Journal of Environmental Economics and Management. 31(3) 269-286.

[Seifert et al.(2008)] Seifert, J., Uhrig-Homburg, M., Wagner, M. 2010. Dynamic Behavior of carbon Spot Prices. Theory and Empirical Evidence. Journal of Environmental Economics and Management. 56(2) 180-194.

[Sikorski(2008)] Sikorski, T. 2008. SpreadTheNews. Trading Carbon Magazine. 2 18-20.

[Tietenberg(1985)] Tietenberg, T. 1985. Emission Trading: an exercise in reforming pollution policy. Resources for the future, Washington D.C. 